\title{
Ueber den Bau und die Thätigkeit der Drüsen.
}

\author{
IV. Mit theilung.
}

Von

\section{Dr. Moritz Nussbaum,}

a. o. Professor und Prosector am anatomischen Institut zu Bonn.

Hierzu Tafel XV-XVIII.

Seit dem Erscheinen meiner III. Mittbeilung hat die Lehre von der Drüsenthätigkeit eine umfassende Bearbeitung durch Heidenhain erfahren. Wie viel noch zu leisten, könnte kaum trefflicher und mit mehr Berechtigung zum Ausdruck gebracht werden, als es im Eingang der Schlussbemerkungen zu Heidenhain's Abhandlung ') geschehen ist. Zwar hatte schon vor Heidenhain im Jahre 1857 Leydig $^{2}$ ) in seiner Histologie auf die verschiedenen Zustände von Drtisenzellen aufmerksam gemacht; auch war im Jahre 1864 Robin durch Cl. Bernard ${ }^{3}$ ) veranlasst

1) R. Heidenhain: Physiologie der Absonderungsvorgänge in Hermann's Handbuch der Physiologie 1880. Bd. $\mathrm{V}$, Theil 1.

2) Leydig: Lehrbuch der Histologie 1857, pag. 317: „Die Zellen, welche bei Batrachiern die Magendrüsen erfüllen, werden in verschiedenen Zuständen getroffen, indem ich bald helle (Landsalamander), bald in verschiedenem Grade körnige beobachtet habe."

3) Cl. Bernard: Du rôle des actions réflexes paralysantes dans le phénomène des secrétions. - Journal de l'anatomie et de la physiologie par Ch. Robin 1864, p. 507, note:

„M. Robin a montré dans ce travail que les deux glandes dont il s'agit diffèrent dans leur structure intime et même que la parotide diffère un peu des sousmaxillaires. Ces glandes présentent, de plus, quelques modifications de structure, relatives en particulier à leur épithélium, suivant qu'on les observe pendant l'état de sécrétion active, ou dans les intervalles de celle-ci à l'état de repos." 
worden, Speicheldrüsen vor und nach der Reizung mikroskopisch zu untersuchen. Trotzdem wird man Heidenhain für den Begründer einer Methode halten miissen, die den Gedanken von dem beständigen Wechsel der Gestalt alles Organisirten und die Vorstellung von der intimen Verknlipfung der Gestaltänderung mit dem Wechsel der Function auf den Bau und die Thätigkeit der Drüsen fruchtbringend anwendet.

Seitdem sind noch nicht zwei Decennien verflossen und wir befinden uns in der That, wie Heidenhain sagt, erst in den Anfängen. - Wenn in Folge dessen die Werthschätzung des Gewonnenen, die Abweisung entgegengesetzter Ansichten zuweilen mit mehr Eifer und Heftigkeit betrieben wird, als es bei völliger Klärung der Probleme denkbar ist, so wird man, den jugendlichen Entw̧icklungszustand unserer Kenntnisse berieksichtigend, die Erklärung und Entschuldigung hierfür finden.

Die vorliegende Mittheilung will, wie die früheren, Beiträge zur Lösung der höchst verwickelten Fragen uber den Secretionsmechanismus liefern, soweit er vorwiegend mit Hülfe des Mikroskops zu erkennen ist. Um dies in übersichtlieher Folge thun zu können, handelt der

I. Abschnitt: von dem Einfluss natürlicher und künstlicher Reizè auf die Thätigkeit der Drüsenzellen.

II. Abschnitt: von der Regeneration der Drüsenzellen im Vorderdarm der Wirbelthiere; zugleich ein Beitrag zur Lehre vom Bau der Magenschleimbaut.

III. Abschnitt: von der Bedentung der Drüsenzellen und den Beziehungen dieses Vorganges zur Secretion.

IV. Abschnitt : von der Veränderung der Kerne und rom Nebenkern der Drïsenzellen.

\section{Abschnitt.}

Von dem Einfluss natürlicher und kinstlicher Reize auf die Thätigkeit der Drüsenzellen.

In der vorigen Mittheilung (dies Archiv, Bd. 16 p. 543) war am Schluss des die Magendrüsen betreffenden Abschnittes gesagt: ,ich halte fest an dem Satz, dass durch den Reiz der eingefuihrten 
Nahrung der Fermentreichthum in den Drüsen gesteigert werde.“ Auf die Kritik, von Grützner an den diesem Satze zu Grunde liegenden Versuchen geübt, möchte ich nicht gern zurückkommen, da wir Beide den Mängeln der Methoden Alles das zur Last legten, was fast ausschliesslich auf die geringe Zahl der Versuche zurïkzufuhhren ist, auf das Untersuchungsobject und auf die verschiedenen Zeiten, zu denen die Versuche am Thier angestellt worden sind. Leider stand mir zu meinen früheren Experimenten keine grosse Zahl von Hunden zu Gebote. Bei den von mir genauer untersuchten Amphibien ist die Secretionsphase eine sehr langdauernde und nicht allein fïuher von mir, sondern bisher iberhaupt nicht vollständig geprüft worden. Meine an Amphibien durch zahlreiche Versuche gewonnenen Resultate glauhte ich unbedingt auch auf die Säugethiere iubertragen zu dürfen, weil erstens auf beiden Seiten eine totale Uebereinstimmung sich mir gezeigt hatte, und weil die Methode Grïtzner's keine exakte ist ${ }^{1}$ ). Die Methode reicht aber ans, um so grosse Unterschiede, wie sie regelmässig vorkommen, augenfällig zu demonstriren.

Fortgesetzte Untersuchungen haben mir nämlich gezeigt, dass sowohl der von Grützner anfgestellte Satz (während der Ruhepause wird Secretionsmaterial in den Drtisenzellen aufgehäuft), als auch der von mir vertretene (durch den Reiz der eingefiulhten Nahrung wird in den Drüsen der Fermentreichthum gesteigert) zu Recht bestehe. Nach der Beschreibung meiner neuen Versuche wird es alsdann möglich sein, diesen beiden Sätzen eine allgemeinere Fassung zu geben.

Von \%wölf im August eingefangenen, längere Zeit hungernden kräftigen Rana esculenta wird ein Exemplar sofort getödtet, ein anderes ohne Nahrung gelassen, den übrigen zehn je ein Muskelstück von dem Gewicht eines Gastrocnemius des getödteten Frosches in den Magen eingeführt.

Diese zehn Frösche wurden darauf in verschiedenen Intervallen getödtet. Da nach zwei Tagen gewöhnlich der Froschmagen nach einer reichlichen Mahlzeit wieder leer geworden ist, so wurden von der 52. Stunde an zwei Frösche gleichzeitig getödtet. Von diesen zwei war der eine kürzere oder längere Zeit vor der Tödtung noch einmal gefüttert worden. An dem gefütterten

1) cf. Hoppe-Seyler: Physiologische Chemie. II. Theil, p. 229. 
Exemplar konnte die Wirkung der durch die eingeführte Nahrung verursachten Reizung mit dem Hungerzustande verglichen werden. Als Maassstab für den Reichthum an Ferment oder dessen nächste Voretufen wurde neben wenigen Verdauungsversuchen vorzugsweise der Gehalt jener grossen Granula in den Oesophagealdrüsen verwerthet, wie sie Bd. XIII dieses Archives beschrieben sind. Man hat nur darauf zu achten, die Granula nicht ausschliesslich frisch untersuchen zu wollen, odler nur an Osmiumsäurepräparaten; da frisch die peripher in den Zellen zu gewissen Zeiten auftretenden Fettgranula kaum von den Fermentkörnern zu unterscheiden sind. In Ueberosmiumsäure werden die durch Aether extrahirbaren, peripher gelegenen Fettpartikelchen intensiver geschwärzt als die Fermentgranula, diese aber während einer bestimmten Periode der Ruhepause in Ueberosmiumsäure völlig gelöst, worauf wir später noch einmal zuriickkommen werden.

Durch Langley's nenere, sowie durch meine früheren Versuche ist die Berechtigung, den Reichthum an Granula mit dem Reichthum an Ferment oder seinen directen Vorstufen zu identificiren, hinlänglich dargethan.

Bei den während des Hungerzustandes gefiutterten Fröschen hatten nun ausnahmelos die Granula in den Oesophagealdrisen wenige Stunden nach der Fütterung zugenommen.

Verfolgt man andererseits den Gang der cyclischen Verände-

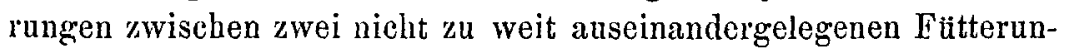
gren, so findet man die Beobachtungen Grützner's am Hund und die Langley's an verschiedenen Amphibien bestätigt: das durch die Verdauung verbrauchte Ferment wird während der Ruhepause von Neuem gebildet. - Fïr den Frosch gelten beziuglich der Pepsin bereitenden Oesophagealdriisen folgende Daten:

Geht man von dem Zeitpunkt des grössten Fermentreichtbums dieser Drisen aus, so wird die Hauptmasse des Secretionsmaterials binnen 48 . Stunden nach einer Fiitterung verbraucht. Von da an steigt der Vorrath an Ferment wieder, der un die 72. Stunde nach der Fitterung in Form selbst in Ueberosmiumsäure löslicher Granula vom Lumen der Alveolen beginnend, bis gegen die Membrana propria hin in den Zellen abgelagert wird. Nach diesem Zeitpunkt werden die Granula in Ueberosmiumsäure unlöslich und das Maximum an Vorrath dieser Granula ist um die 96. Stunde vorhanden. Es umfasst somit die ganze Phase einer Secretion bei den Oeso- 
phagealdrüsen des Frosches (Rana esculenta) volle vier Tage. Diese Zeit kann durch eine friiher eintretende Futternng abgekürzt werden, indem schon nach zwei Tagen die eingefubrte Nahrung die ganzen Zellen mit Granula füllt. Es kann dagegen auf dem von der Natur gegebenen Culminationspunkt der Aufspeicherung von Secretionsmaterial, also am vierten Tage, durch Nahrungszufuhr keine weitere Steigerung des Vorrathes an Ferment erzielt werden. Demgemäss ist die Curve der Bildung des Secretionsmaterials und der Ausstossung des Secretes in den Oesophagealdrüsen von Rana esculenta auf die Zeit von vier Tagen berechnet; ihre Gestalt kann durch künstliche Nahrungszufuhr verändert werden, indem der aufsteigende Schenkel durch eine Fütterung vor dem vierten Tage steiler wird.

Der Uebersichtlichkeit halber und im Interesse einer kürzeren Darstellung folgen die Resultate einer Serie von Versuchen am Frosch (Rana esculenta) in Form einer Tabclle (s. pag. 301).

Fiitterungsversuche an Salamandra maculosa, wesentlich zum Zweck des Studiums der Regeneration der Drüsenzellen angestellt, ergaben gleiche Resultate. Die Entleerung des Magens nimmt 72 Stunden und mebr in Anspruch. Nach längerem Hungern kann man binnen 8 Stunden die aus den Labdrisen und dem Pancreas während der Hungerperiode fast völlig geschwundenen Granula durch Nalrungszufuhr wieder reichlich anhäufen; die Drïsen entleeren das Secretionsmaterial im Laufe der Verdauung und bilden es vom vierten Tage nach einer Fütterung wieder neu in den Zellen.

Bei hungernden Salamandern und auch vielen anderen Amphibien ist nicht selten in dem sonst leeren Magen die abgestossene Epidermislage des Thieres aufzufinden.

Um die 72. Stunde nach der Futterung enthalten die Labdrüsen peripher in den Zellen reichlich Fettkörnchen; eine Erscheinung, wie sie von den Fileiterdrüsen der Amphibien nach Ablauf des Laichgeschäfts und auch von den Oesophagealdrüsen und Labdrüsen des Frosehes bekannt ist.

Electrische Reizung der einzelligen Drïsen von Argulus foliaceus bewirkte Entleerung des angehäuften Secretionsmaterials, das in der Ruhepause längstens in drei Tagen wieder völlig neugebildet wurde.

Reizung der Schleimdrüsen in der Haut von Salamandra ma- 
Ueber den Bau und die Thätigkeit der Drüsen.

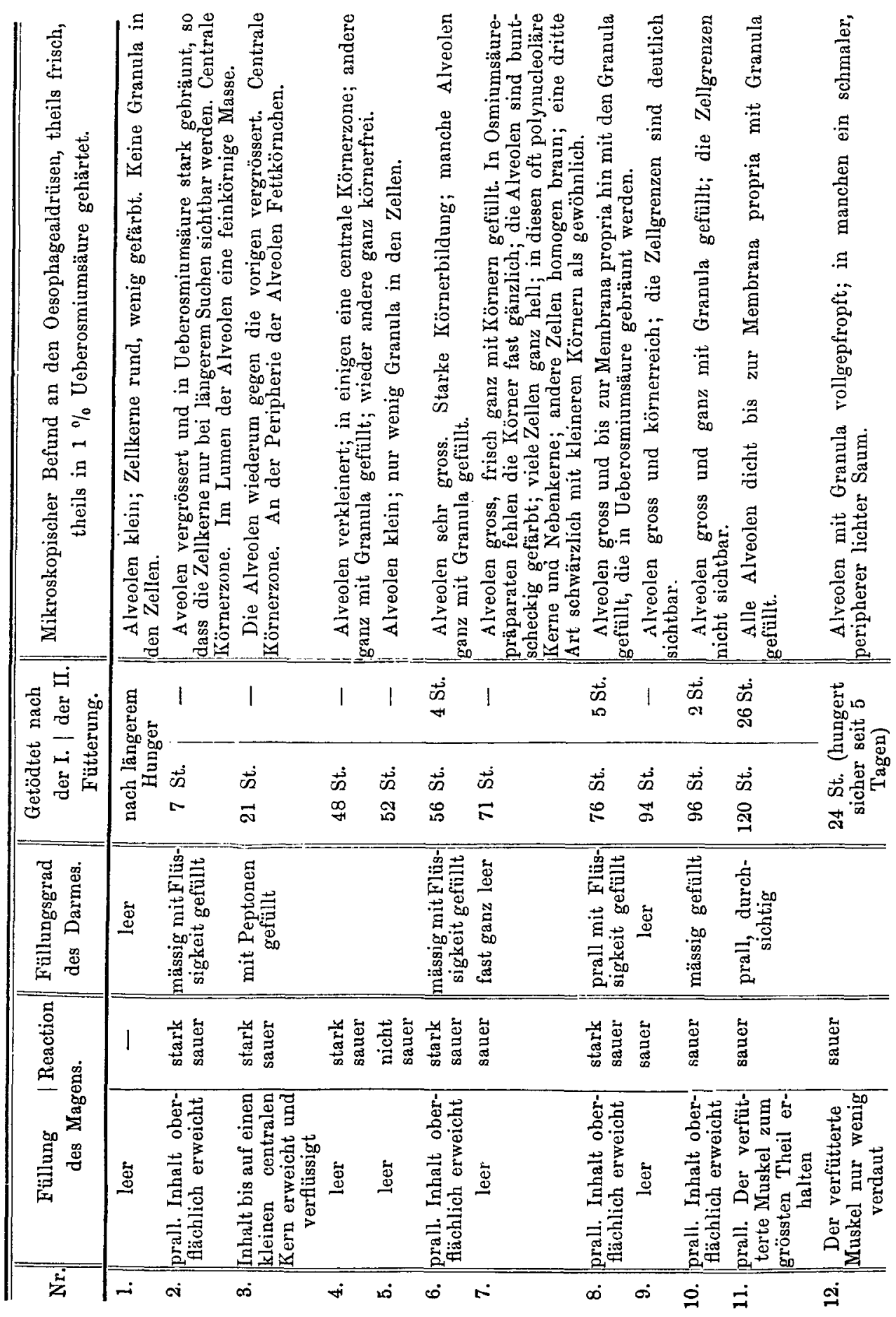


culosa ") brachte eine Entleerung des Schleimes zu Wege. Die schleimigen Köpfe der Zellen verschwanden durch die Reizung: indem sie als Secret verflüssigt und entleert wurden. Die Kerne der Zellen werden nach der Reizung grösser, wie dies in höchst auffallender Weise auch an den Eileiterdrüsen der Amphibien zu verschiedenen Jahreszeiten beobachtet werden $\mathrm{kann}^{2}$ ). Wenn der Schleim aus den Zellen dieser Drüsen entleert ist, werden die zur Zeit der reichlichsten Anfüllung der Zellen mit Schleim kaum sichtbaren Kerne wieder saftig und gross. Dasselbe ist ja auch von Zellen bekannt, die Reservestoffe, wie Fett, Stärke etc. in sich aufnelumen und wieder abgeben können.

Fassen wir die gewonnenen Resultate zusammen, so gilt für fermentirende und schleimbereitende Drüsen der auch von He i denhain ausgesprochene Satz, dass in der Ruhepanse Secretionsmaterial in den Zellen angehäuft und bei dem Act der Absonderung in die definitiven Secretbestandtheile übergeführt werde. Für jede Drüse jedes einzelnen Thieres besteht eine bestimmte Phase, die beirn natiirlichen Ablauf der die Secretion bedingenden Vorgänge streng inne gehalten wird. Es ist aber auch möglich, durch künstlich eingefïhrte Reize Secretionsmaterial in den Zellen anzuhäufen zn Zeiten, wo ohne den Reiz die Zellen nach wie vor arm an specifischen Bestandtheilen geblieben wären. Die Nahrungszufuhr bei länger bungernden Thieren regt nicht allein die Ausstossung, sondern auch die Bildung der Secretionsstoffe so mächtig an, dass die Bildung bei Weitem den Verbrauch iibertrifft, was bei Zufuhr der Nabrung am Ende der natürlichen Phase keineswegs der Fall ist. Dann verarmt die Drise progressiv an Secretionsmaterial. Nahrungszufuhr beschleunigt aber auch die Bildung der Secretbestandtheile in den Drïsenzellen, wenn sie in eine Zeit fällt, wo unter natiirlichen Bedingungen die Vorstufen der Secrete noch nicht in den Zellen regenerirt sind.

1) Zum bequemen Vergleich der gereizten und ungereizten Drüsen wird man folgende Einrichtung des Versuchs geeignet finden: Man decapitirt einen. Salamander, schneidet die Wirbelsäule mit Haut und Rückenmuskeln aus dem Rumpf heraus und führt in kurzem Abstande nadelförmige Electroden quer durch das Präparat. Zwischen den Electroden bedecken sich nach jeder Reizung die Oeffnungen der kleinen Schleimdrüsen mit weissen Tröpfchen; die übrige Haut bleibt trockeu.

2) Hebold: Ein Beitrag zur Lehre von der Secretion und Regeneration der Schleimzellen. Inaug. Dissert. Bonn 1879, p. 29. 


\section{Abschnitt.}

Von der Bedeutung der Drïsenzellen im Vorderdarm der Wirbelthiere; zugleich ein Beitrag zur Lehre vom Bau der Magenschleimhaut.

Wie in dem vorigen Abschnitt die Widerspritche in Bezug auf den Modus der Secretbildung eine befriedigende Lösung erfahren haben dürften, so glaube ich auch durch das Folgende einen Beitrag zu liefern, der den lang gefuihrten Streit um die Natur der Haupt- und Belegzellen beizulegen im Stande sein wird.

Die von mir als Reagens auf Fermente empfohlene Ueberosmiumsäure leistet nicht genug. Dass sie jedoch wirksame Fermente intensiv bräune, ist nicht in Frage zu ziehen. Wenn sie auch andere Körper bräunt oder schwärzt, so kann dies keinen Gegengrund für ihre Anwendung abgeben. Die Schwäche der Reaction besteht darin, dass gewisse Vorstufen des Ferments der Labdrüsen in dem Reagens spurlos aufgelöst werden, was ich erst in neuerer Zeit habe erkennen können.

Als Heidenhain in den Labdrüsen der Säugethiere zwei Zellenarten, die Haupt- und Beleg-Zellen, entdeckt hatte und Ebstein die Hauptzellen mit den Zellen der Pylorusdrüsen identificiren zu müssen glaubte, nahm man, gestützt auf die von Heidenhain und Grïtzner angestellten Verdauungsversuche, ziemlich allgemein an, dass die Pylorusdrisen sowie die Hanptzellen der Labdrùsen das Ferment, und dass die Belegrellen die Säure des Magensaftes producirten. Es gibt, so folgerte man, zwei Drüsenarten im Magen der Säugethiere: die Haupt- und Belegzellen führenden Labdrüsen und die ausschliesslich Hauptzellen tragenden Pylorusdrüsen.

In Hermann's Handbuch der Physiologie hat Heidenhain auf einige Unterschiede im feineren Bau der Hauptzellen und Pylorusdrisenzellen anfmerksam gemacht; aber erst Langley wies nach, dass zwischen diesen beiden Zellenarten keine Uebereinstimmung bestehe.

Die Entdeckung Heliodor's von Swiecicki, dass in den Oesophagealdriisen der Frösche Pepsin gebildet werde, ist nach verschiedenen Richtungen verwerthet worden. Heidenhain and seine Schüler, sowie Langley fanden in der Uebereinstimmung 
der frischen Oesophagealdrüsenzellen des Frosches und der von ihnen untersuchten Haupt\%ellen der Säugethierlabdrtisen, einen neuen Beweis für die Pepsinbildung in den Hauptzellen der Säugethierlabdrüsen. Ich selbst glaubte wegen der Resistenz und Bräunung der Granula der Oesophagealdrüsenzellen des Frosches und der Löslichkeit der Granula der Hauptzellen in Ueberosmiumsäure jede Beziehung zwischen beiden Zellenarten von der Hand weisen zı können. Es schien nicht wahrscheinlich, dass ein und derselbe Körper das eine Mal in einem Reagens löslich, das andere Mal darin unlöslich sein sollte.

Wie schon oben mitgetheilt, sind aber auch zu gewissen Zeiten die Granula in den Oesophagealdrüsen der Frösche in Ueberosmiumsäure löslich. - Dië neueren Publicationen Langle y's berichten von Unterschieden der Löslichkeitsverhältnisse bei den Granula in den Hauptzellen verschiedener Säugethiere. Es wird unten gezeigt werden können, dass auch bei den Säugethieren kein Unterschied in der Species, wie Langley anzunehmen scheint, sondern einfach der Zeit nach existirt, ob die Fermentyranula. der Hauptzellen in Ueberosmiumsäure löslich sind oder nicht.

Die Oesophagealdrüsenzellen des Frosches und die Hauptzellen der Säugethierlabdrüsen enthalten grosse Körner, die erst in Ueberosmiumsäure löslich sind, später aber in diesem Reagens erhalten bleiben und gebräunt werden. Die Körner sind in den gebräuchlichsten mineralischen und organischen Säuren und in Alkalien löslich. Die Zellen füllen sich mit den Körnern, dem Secretionsmaterial, von dem am Lumen der Schläuche gelegenen Theile aus und kommen somit in diesem Punkte völlig mit den Zellen des Pancreas überein.

In Folge dieser Uebereinstimmung muss ich meine frühere Behauptung zurücknehmen, das Ferment sei ausschliesslich in den Belegzellen vorhanden.

Die Hauptzellen bilden also Ferment, wie Heidenhain, seine Schiiler, sowie Langley behaupteten. Der Reichthum an Körnern in den Hauptzellen gibt auch hier einen Maasstab ab für. die Schätzung des Fermentgehalts, wie ich dies früher von den Oesophagealdrüsen der Frösche angegeben hatte.

Wo man das Secret der Belegzellen isolirt auffangen konnte, wie beim Frosch, fand man ein saures sofort wirksames Pepsin darin. Das von den Hauptzellen (Oesophagealdrüsen des Frosches) 
zu gewinnende Secret ist alkalisch und bedarf zu seiner Wirksamkeit erst des Zusatzes von Säure.

Meine fruher am Frosch angestellten Versuche, den Magen $\mathrm{zu}$ isoliren und das von seinen Belegzellen gelieferte Secret gesondert auf Eiweisskörper einwirken zu lassen, habe ich zwar selbst nicht für völlig beweisend erklärt. Diese Versuche werden aber durch die von Lan gley kürzlich veröffentlichten Verdauungsversuche hinlänglich bestätigt.

Die Belegzellen in den Labdrtisen der Säugethiere und der Frösche sind fein granulirt; sie bräunen sich in Ueberosmiumsäure und liefern eiweissverdauendes Ferment in saurer Lösung.

Die Pylorusdrtisenzellen sind Schleimzellen; sie bräunen sich weder in Ueberosmiumsäure, noch liefern sie peptisch wirksame Extracte. Es kommen aber bei manchen Säugethieren Belegzellen in den Pylorusdrïsen vor, die von mir schon früher für die Möglichkeit der Gewinnung peptisch wirksamer Extracte aus der Pylorusschleimhant des Hundemagens verantwortlich gemacht worden waren.

Diese drei Arten von Drüsenzellen: Haupt-, Beleg- und Pylorusdrüsenzellen kommen allen Wirbelthierklassen gleichmässig zu; wenn wir von den Säugethieren abseben, so ist selbst die Reihenfolge und die Form der Drïsen, in denen die obengenannten Zellen sich finden, eine typische und für alle Klassen gemeinschaftliche.

Rechnet man zu diesen Drüsenformen noch die in ihrem Vorkommen höchst inconstanten Schleimdrỉsen des Oesophagus hinzu, so trägt die Schleimhaut des Vorderdarmes der Wirbelthiere der Reihe nach

1. Schleimdrtisen des Oesophagus.

2. Zusammengesetzte Pepsindrüsen, ausschliesslich Hauptzellen führend; Secret (beim Frosch geprlift) alkalisch.

3. Einfache Pepsindrtisen, ausschliesslich Belegzellen fithrend; Secret (beim Frosch geprüft) sauer.

4. Schleimdrüsen des Pylorus.

Diese Drtisen können in verschiedener Weise auf Oesophagus und Magen. vertheilt sein; bei Rana liegen die sub 2 angefuhrten Drüsen im Oesophagus; bei Lacerta an der Grenze von Oesophagus und Magen; bei Triton im ersten Abschnitt des Magens.

Für die Amphibien hat Langley eine ähnliche Eiutheilung 
gegeben; nur wird man die Uebereinstimmung in Bau und Function seiner Oesophageal glands und anterior oxyntic glands nicht läugnen können.

Die Säugethiere haben Haupt- und Belegzellen in denselben Drüsenschläuchen gemischt; neben den Schleimdrüsen des Oesophagus kämen hier also nur noch zwei Drüsenarten, Lab- und Pylorusdruisen vor. Doch gibt es auch Säugethiere, wo wenigstens der Form nach drei Drüsenarten in derselben Reihenfolge wie bei den übrigen Klassen unterschieden werden können: auf die grosse zusammengesetzte Magendrise des Biber (Castor fiber) folgen einfache Pepsindrïsen und dann ein Pylorustheil.

Zur Illustration des Gesagten mögen einige mehr oder weniger detaillirte Beschreibungen der Schleimhaut des Vorderdarmes verschiedener Repräsentanten aller Wirbelthierklassen folgen.

\section{Der Magen der Säugethiere.}

Bei den Säugethieren ist die Histologie der Magendrüsen bis jetzt nur auf wenige Species ausgedehnt worden. Soweit die vorbandenen Beobachtungen einen Schluss gestatten, scheint eine und dieselbe Anordnung allgemein vorhanden zu sein. Die Driisen sind einfach oder zusammengeset»t schlauchförmig; sie nehmen von der Mitte des Magens aus nach auf- und abwärts, sowobl gegen die Cardia als den Pylorus zu allmählich an Länge ab. Die Schleimbaut gliedert sich in zwei grössere Abschnitte: die Labdrüsen- und Pylorusschleimdrüsenzone. Dabei ist jedoch ein Unterschied bei verschiedenen Thiergruppen zu constatiren, indem bei manchen der Pylorustheil bedeutender, bei anderen geringer ausgebildet ist. Dies erkennt man makroskopisch an der mächtigen Muskulatur des Pylorustheiles und der hellen dünnen Schleimhaut dieser Zone. Die Region der Lajdrïsen hat dïnne Muskulatur, dicke chocoladenfarbene Schleimhaut. - Bei den Wiederkäuern bezieht sich das Gesagte selbstverständlich nur auf den Labmagen. Beim Pferd kommt im Ánfangstheil des Magens noch ein grosser, drüsenloser Blindsack hinzu. Viele Nager haben ausser den beiden genannten Abschnitten noch eine besondere Magendrise.

Ueber den feineren Bau der Pylorusschleimhaut der Säugethiere ist nur wenig zu sagen. Die Drüsen sind ächte Schleim- 
drüsen. Kurzlich hat Stöh $\mathrm{r}^{1}$ ) auch beim Menschen Belegzellen ${ }^{2}$ ) dort nachgewiesen und seinen Fund durch treffliche Abbildungen illustrirt. Auch der Pylorustheil des Fledermäusemagens enthält vereinzelte Belegzellen. Beim Maulwurf ist die Ausdehnung der Pylorusschleimdrisen eine ganz minimale.

Eine eingehende Durchmusterung der eigentlichen Labdrisenzone verschiedener Säugethiere hatte Langley ${ }^{3}$ ) dazu geführt, zu unterscheiden zwischen solchen Arten, bei denen die Granula der Hauptzellen in Ueberosmiumsäure erhalten bleiben und solchen, wo durch Einwirkungen von Ueberosmiumsäure die Granula aus den Hauptzellen spurlos verschwinden. Es wird gezeigt werden können, dass dieser Unterschied nicht besteht (cf. oben p. 304).

Ausser den Haupt- und Belegzellen kommen den Labdrüsen aller Säugethiere noch jene von Heidenhai ${ }^{4}$ ) zuerst beschriebenen „kleineren Zellen“ zu. Sie finden sich im Drlisenhalse und in oheren Ende des Drüsenkörpers am zahlreichsten, also dort, wo die ausgeprägten Hauptzellen selten werden oder gänzlich verschwinden.

Bei vielen Säugethieren, am prägnantesten wohl bei den Fledermäusen, sitzen die Hauptzellen am Grunde der Scbläuche und die Belegzellen, sowie die kleinen Zellen in oberen Theile derselben. Bei den meisten Säugethieren jedoch sind Haupt- und Belegzellen völlig gemischt.

Die absolute Länge der Drïsenschläuche variirt nach der Species, abgesehen von den rclativen Unterschieden der einzelnen Localitäten der Schleimhaut. Hund, Schwein, Kaninchen haben lange Labdrüsen; Meerschweinchen, Maulwurf, Fledermaus kurze.

Das Cylinderepithel der Oberfläche geht versehieden weit in die Schläuche nach abwärts; im Allgemeinen um so tiefer, je näher dem Pylorus. Oft sitzen Belegzellen dicht an Ausgange der Drüsen.

1) $\mathrm{Ph}$. Stöhr, d. Arch. Bd. 20, Taf. 15.

2) Die Belegzellen im Pylorustheil des Hundemagens sind von mir (d. Arch. Bd. XVI) beschrieben worden. Die von Grützner gegen die Belegzellennatur dieser Zellen erhobenen Einsprüche sind schon von Stöhr (1. c.) widerlegt worden.

3) Langley: Journal of Physiology. Vol. III. Nr. 3.

4) Heidenhain, d. Arch. Bd. VI, p. 389. 
Die Hauptzellen des Hundes, des Kaninchens, der Fledermaus sind gross und deutlich gegen die Belegzellen abgehoben; die Hauptzellen des Maulwurfs and des Meerschweinchens sind klein.

Während des Hungerzustandes sind nach Heiden hain die Hauptzellen gross und die Belegzellen klein. Während der Verdauung gilt das umgekehrte Verbältniss.

Wie Heidenhain ${ }^{1}$ ) angibt, dauert beim Hunde, wenn derselbe durch 24stiundige Nahrungsentziehung auf eine reichliche Mahlzeit vorbereitet worden ist, die Verdauung bis zur völligen Entleerung des Magens gegen 20 Stunden.

Der Maulwurf hat nach 1 bis 2 Stunden seine nach längerem Hunger aufgenommene Nahrung völlig verdaut.

Es ist bekannt, dass Kaninchen des Hungertodes sterben können, ohne ihren Magen von Speiseresten vollständig entleert zu haben.

Das Meerschweinchen ist weit gefrässiger als das Kaninchen und hat schon 10-12 Stunden nach der Nahrungsaufnahme seinen Magen entleert.

Vergleicht man mit diesen Daten den relativen Gehalt der Magenschleimhäute dieser Thiere an Hauptzellen, so weisen die Thiere mit langsamer Verdauung einen grösseren Reichthum dieser Zellenart auf, während bei dem gefrässigen und schnell verdauenden Maulwurf und dem Meerschweinchen die Belegzellen vorwiegen. Ob diese Unterschiede durchgreifend seien, muss durch weitere Untersuchungen erst bestätigt werden.

Die hier folgenden Notizen über die Schleimhaut des Magens einiger Säugethiere machen keinen Anspruch auf erschöpfende Behandlung aller in Frage kommenden Details; sie sind gelegentlich von Beobachtungen gewonnen, welche auf die Veränderung von Zellleib und Zellkern durch die Secretion gerichtet waren.

$$
\begin{gathered}
\text { Der Magen des Kaninchens }{ }^{2} \text { ) (Lepus cuniculus). } \\
\text { Figg. 1, 2, 11, } 21 .
\end{gathered}
$$

Die Magendriisen des Kaninchens sind ron Langle y und Sewall in neuerer Zeit noch beschrieben worden, so dass dieser

1) Handbuch der Physiologie von Hermann. V. Bd., 1. Theil, p. 142.

2) Vergleiche hierzu: Roll ett, Untersuchungen aus dem Institute für Physiologie und Histologie in Graz. II. Heft. Heidenhain 1. c.; Langley and Sewall: Journal of Physiology Vol. II. Nr. 4, p. 293. 
Darstellung nur Weniges hinzuzufügen bleibt. Von der Ausdehnung des Pylorustheiles an der kleinen Curvatur bis gegen die Cardia findet man bei Langley und Sew all eine schematische Darstellung. Die Angaben iber die Vertheilung der Granula in den Hauptzellen der verschiedenen Regionen des eigentlichen Labdrüsentheils und die Vermehrung dieser Granula nach kurzer Hungerperiode kann man schon makroskopisch erkennen. Die Drüsen des Fundus sind bei hungernden Thieren weiss, bei verdauenden durchsichtig; die Drüsen der grossen Curvatur findet man fast durchweg hell und durchscheinend. Bei mikroskopischer Untersuchung bestätigt es sich, wie Langley und Sewall angeben, dass die Hauptzellen der Fundusdrusen beim bungernden Thier mit glänzenden Granulis angefüllt sind. Die Granula sind gross, girösser als andere unzweifelhafte Fermentgranula desselben Thieres und lösen sich in Ueberosmiumsäure (cf. oben pag. 304). Rollett und Heidenhain haben diese Granula der Hauptzellen wohl gekannt; auch wird man sich zur bequemen Untersuchung des frischen Objectes wohl der Angabe Rollett's von der leichten Isolirbarkeit der Labdrtisenschläuche des Kaninchens erinnern. Zur bequemen Demonstration frischer Magendrüsen und ibrer beiden Zellenarten ist das Kaninchen am rneisten zu empfehlen.

Setzt man zu den in Jodserum isolirten Schläuchen einen Tropfen Ueberosmiumsäure $z u$, so erhält man Präparate, die in Glycerin eingeschlossen dauernd und prägnant die Unterschiede zwischen Haupt- und Belegzellen, zwischen den Drüsen im Fundus des Magens und an der grossen Curvatur erhalten. Die Fundusdrüsen sind kürzer als die in der Mitte der grossen Curvatur. Im Fundus hungernder Thiere erscheinen die Hauptzellen gross, hell und mit dunkel gefärbten zackigen Kernen; die Belegzellen sind gebrïunt, scharf contourirt, fein granulirt; ihre Kerne gross, blass, oval, mono- bis polynucleolär. In der grossen Curvatur verschwinden die Färbungsunterschiede mehr, da auch die Hauptzellen einigermaassen gebräunt sind; die Kerne derselben haben stets ein Kernkörperchen. In der nächsten rechts gelegenen Umgebung der Cardia sind Haupt- und Belegzellen in den Schläuchen gemischt; dann beginnt die Formation der Pylorusdrüsen, deren Inbalt in verdünnter Essigsäure sich tribt, während die Labdrüsenschläuche durch dieses Reagens aufgehellt werden.

Ob die Belegzellen an der grossen Curvatur, wie Langley Archiv f. mikrosk. Anatomie. Bd. 21. 
angibt, überwiegen, während im Fundus die Hauptzellen zahlreich sind, dürfte schwer zu entscheiden sein.

Es gibt an der grossen Curvatur Schläuche, die in ibrem unteren Ende auch nicht eine einzige Belegzelle enthalten. Prägnant sind allein die Unterschiede der Hauptzellen beider Regionen im Gehalt an Granula und mit Bezug auf die Form ibrer Kerne, namentlich nach Erhärtung in Ueberosmiumsäure. Da jedoch die Kerne in den Hauptzellen der Fundusdrisen im unveränderten Zustande durch die groben Granula verdeckt werden nnd erst za erkennen sind, wenn die Granula gelöst wurden, so kann nicht gut entschieden werden, in welcher Weise das chemische Reagens bei der Lösung der Granula auch auf die Kerne eingewirkt hat.

\section{Der Magen des Meerschweinchens ${ }^{1}$ ) (Cavia cobaya).}

Der weissliche Pylorustheil bildet eine gürtelförmige Zone, auf die etwa der sechste Theil der Länge des ganzen Magens kommt; der tibrige Theil der Schleimbaut ist grauröthlich gefürbt und mit Labdrüsen besetzt. Im Fundus des Magens messen die Schläuche durchschnittlich $0,2 \mathrm{~mm}$, an der grossen Curvatur $0,35 \mathrm{~mm}$, doch so, dass sie nach dem Pylorus zu an Länge wieder abnehmen. Die Drüsen sind frisch in Jodserum oder öligen Fllissigkeiten gut zu isoliren. Tödtet man Thiere nach etwa 12stündigem Hunger, so erscheinen die Labdrüsen wie dunkele mit hellen Buckeln beschlagene Stäbe. Das Centrum der Schläuche bildet eine unregelmässig begrenzte Zone grosser glänzender Granula, die in verdünnten Säuren (Salzsäure, Essigsiiure, Ueberosmiumsïure) und Ammoniak spurlos verschwinden. Langley gelang es, diese Granula in Ueberosmiumsäure zu conserviren ${ }^{2}$ ). Vielleicht haben wir auch an diesem Object nicht zu denselben Zeiten untersucht und es ist denkbar, dass die lösliche Form der Granula in eine unlïs-

1) Vergleiche hierzu: Rollett: Untersuchungen aus dem Institute für Physiologie und Histologie in Graz. II. Heft, p. 171. - Langley: Journal of Physiology. Vol. III, Nr. 3.

2) Nachträglich fïge ich hinzu, dass ich auch beim Meerschweinchen in Ueberosmiumsäure unlösliche Granula der Hauptzellen, wie Langley angegeben, gefunden habe; es bestätigt sich also die oben ausgesprochene Vermuthung. 
liche übergeführt wird oder umgekebrt. Damit würden auch die Angaben Langley's (l. c. pag. 273) vom Magen des Kaninchens, des Frettchens und des Schafes tibereinstimmen, wo sich zuweilen die Granula der Hauptzellen in Ueberosmiumsäure erbalten, gewöhnlich aber darin verschwinden. Untersucht man gleichzeitig die Zellen des Pancreas von demselben Thiere, so sind die Granula in den Zellen dieser Drüse kleiner als die der Hauptzellen und etwa von derselben Grösse, wie die Granula der Belegzellen. Die Granula der Belegzellen sieht man frisch und nach Erhärtung in Ueberosmiumsäure recht gut. Zusatz von Essigsäure macht die Belegzellen kurz nach der Einwirkung des Reagens ganz glasig; bald darauf werden die Zellen wieder dunkler und erscheinen grob granulirt. Etwas Aehnliches berichtet Heidenhain ${ }^{1}$ ) von der Einwirkung des sauren Magensaftes: „Hat man bei Anfertigung eines Schnittpräparates Spuren des sauren Magensaftes von der Oberfläche her auf den Drlisengrund übertragen, so heben die Belegzellen sich heller und durchsichtiger gegen den dunkeln Schlauchinbalt ab."

Der Reichthum der Labdrüsenschläuche an Belegzellen steht auf einer Linie mit dem der gleichen Drüsen des Maulwurfs. Waihrend man bei Hund, Schwein, Kaninchen oft recht ansehnliche Strecken fast ausschliesslich mit grossen Hauptzellen bekleidet findet, sind die gleichwerthigen Zellen in Labdrüsenschläuchen des Meerschweinchens klein und nicht zahlreich. An Längsschnitten in Alkohol oder Osmiumsäure erbärteter Schleimhautstiicke sehen die in Anilinfarben tingirten Schläuche aus, als wären sie nur aus Belegzellen zusammengesetzt. Stärkere Vergrösserungen lassen eine feine bandartige, stärker gefärbte Zeichnung zwischen den Belegzellen erkennen. Dies sind die Hauptzellen, deren Contouren man am besten auf Querschnitten ansichtig wird.

Der Magen der Fledermans ${ }^{2}$ ) (Vesperugo Nathusii und Vesperugo serotinus). Taf. XV. Figg. 3, 5, 12.

Der Magen der untersuchten Species ist dünn und durchscheinend. Im Fundus sind die Driisenschläuche kurz: sie werden

1) Heidenhain: Archiv für mikrosk. Anatomie, Bd. VI, p. 376.

2) Vergleiche hierza: Rollett l. c. - Langley l. c. 
bis gegen die Mitte der grossen Curvatur länger und nehmen zum Pylorus hin wieder an lïnge ab. Belegzellen finden sich wie beim Hunde und Menschen in allen Magendrisen, und zwar sind die spärlichen Belegzellen in den Schleimdriisen des Pylorus in keiner Weise von den Belegzellen der übrigen Drüsenschläuche verschieden. Bei hungernden Thieren - es fanden sich bei einem frisch eingefangenen Exemplare einige Haare, bei dem anderen einige unverdauliche Reste im Magen vor - sind die Hauptzellen vieler, doch nicht aller Fundusdrisen mit groben Körnern angefüllt; diese groben Körner fehlten den Hauptzellen in den Drüsen an der grossen Curvatur, wie es auch Langley beschrieben hat. Die Belegzellen sind dunkel und fein granulirt, ihre Kerne zuweilen polynucleolär.

Safranin färbt die Granula der Hauptzellen sehr intensiv, so dass bei geeigneter Behandlung auf diese Weise bequem die Vertheilung der Haupt- und Belegzellen in den Schläuchen und die Verbreitung der Granula in den Hauptzellen zo studiren ist. ') Man ïberzeugt sich dann auch, wie schwer es ist, die Gruppirung von Haupt- und Belegzellen schematisch darzustellen. Oft reichen die Belegzellen bis dicht unter das Oberfächenepithel; oft ist die erste Belegzelle dureh ein recht langes Scbaltstick von der Oberfläche entfernt. Der Drüsengrund der Schläuche des Fundus enthält vorwiegend Hauptzellen; doch sind anch in vielen Fällen Belegzellen eingestreut. Die mittlere Partie der Drüsenschläuche trägt wohl die meisten Belegzellen; nur von wenigen körnerreichen Hauptzellen ist diese fast continuirliche Belegzellengruppe unterbrochen. Es gibt aber auch Schläuche, deren mittlere Partie ziemlich breite Ringe von continuirlich aneinandergefugten Hauptzellen enthält. Im Drïsenhalse sind zwischen den in Osmiumsäure gebräunten Belegzellen helle, kleinere Zellen mit ovalem oder rundem Kern und grossem glänzenden Kernkörperchen eingeschaltet.

Die Granula der Hauptzellen nebmen in den meisten Fällen blos die centrale Partie der Zelle ein; oft ist die ganze Zelle damit erfüllt.

1) Wo die Granula sich nicht in Osmiumsäure conserviren, heben sich bei kurzer Einwirkung von Anilinfarben die Hauptzellen ebenfalls deutlich $a b$, wie Rollett dies bereits beschrieben bat. 


\section{Der Magen des Manlwarfs ${ }^{1}$ ) (Talpa europaea)}

Figg. 4, 6, 23.

zeichnet sich durch seine Durchsichtigheit vor dem anderer Säugethiere wesentlich aus. Die Drüsen sind inselförmig gruppirt, was namentlich gut nach Injection verdünnter Ueberosmiumsäure sichtbar wird. Der Pylorustheil ist auffallend klein und bildet einen Ring von etwa $6 \mathrm{~mm}$ Länge. Seine Drüsen sind niedrig, einfach oder verzweigt schlauchförmig und werden in Ueberosmiumsäure nicht gebräunt. $3 \mathrm{~mm}$ vom Anfang des Duodenum entfernt finden sich vereinzelte Belegzellen in den Drüsenschläuchen.

Von da an nimmt die Zahl der Belegzellen enorm zu. In der eigentlichen Labdrüsenzone findet man nur schwer die kleinen Hauptzellen zwischen den Belegzellen; doch sind sie wie überall vom Fundus der Schläuche bis zun Drüsenhalse zwischen deu Belegrellen gruppirt. Fiittert man ein hungerndes Thier, so sehen die Belegzellen in der oberen Hälfte der Schläuche wie angefressen aus; ihre Kerne sind multinucleolär, während die Kerne der an der Basis gelegenen Belegzellen und die Kerne der Hauptzellen mononucleolär nach Behandlung mit Ueberosmiumsäure erscheinen.

Die Belegzellen sind fein granulirt. In der centralen Hälfte der Hauptzellen an der Basis der Schläuche finden sich zuweilen grössere Granula, die in Osmiumsäure leicht gebräunt werden.

Was das Vorkommen der Belegzellen anlangt, so sind vorhin die nöthigen Angaben darüber schon gemacht worden. - Die Haupt-

1) Vergleiche hierzu: T. Cuvier: Vorlesungen über vergleichende Anatomie, übers. von J. F. Meckel 1810, III. Theil, p. 385. - F. Leydig: Histologie 1857, p. 317. - Langley: Journ. of Physiology. Vol. III, Nr. 3, pag. 270.

Es dürfte vielleicht interessiren, einige Notizen über das Verdauungsvermögen des Maulwurfs hier anzuschliessen.

Ein hungernder Maulwurf verzehrt mit grosser Gier bis zu acht feiste Regenwürmer, deren vorderes Leibescnde er geschickt mit den Vorderfüssen erfasst und dann bissenweise in den Rachen nachschiebt. Ein bis zwei Stunden später ist in seinem Magen kaum eine Spur der genossenen Mahlzeit mehr zu finden. Die Verdauung ist jedenfalls eine sehr energische. Das für einen Carnivoren auffallende Verhältniss der Darmlänge zur Länge des Körpers $(8: 1)$ ist schon von $\mathrm{Cuvier}$ angegeben worden. Am Darme ist äusserlich kein Colon und kein Coecum abgesetzt. Nahe dem Rectum und weiter nach aufwärts zeigt der Darm schöne Peyer'sche Plaques. 
zellen finden sich am Grunde der Schläuche in ausgeprägter Form und mit centraler in Ueberosmiumsäure gebräunter Zone grosser Granula bis nahe am Pylorus. Ein leicht mit Ueberosmiumsäure aufgeblähter Magen eines erwachsenen Maulwurfs misst an der grossen Curvatur von der Kuppe des links gelegenen Blindsackes bis zum Pylorus $10 \mathrm{~cm} ; 1,5 \mathrm{~cm}$ vom Pylorus entfernte Schnitte enthalten noch Hauptzellen.

Die Cylinderepitbelien der Mageninnenfläche sind während der Verdauung von einer mehrschichtigen Schleimlage bedeckt, zwischen deren einzelnen Lamellen eine grosse Zahl zu Grunde gegangener Zellen eingeschlossen ist.

Hat ein Maulwurf zwei bis drei Tage gehungert, so sind die Belegzellen klein; manche enthalten keine Granula, sind aber in Ueberosmiumsäurepräparaten stark gebräunt.

\section{Der Magen des Bibers ') (Castor fiber).}

Der Magen vieler Nagethiere zeigt bekanntlich vom Baue des Säugethiermagens abweichende Eigenthïmlichkeiten. Schon früh ist der Magen des Bibers mit dem Vogelmagen verglichen worden.

Durch die Gite des Herrn Geheimrath von Leydig stand mir der Magen eines Castor fiber zur Disposition. Leider war an dem in diluirtem Alcohol aufbewahrten alten Sammlungspräparate die histologische Ausbeute nur gering. Mit Sicherheit konnte jedoch festgestellt werden, dass die Magenschleimhaut in drei verschiedene Abschnitte zerfalle: 1) die grosse Drüse; 2) daran sich anschliessend und den links gelegenen Blindsack nebst Curvatura major und minor umgreifend eine Zone, in deren Schläuchen viele isolirte Zellen erhalten waren; 3) zuletzt ein Pylorustheil mit völlig macerirten Drüsenschläuchen, von denen nur noch die bindegewebigen Hüllen, das Stroma, restirten. Vergleiche mit ähnlich behandelten, aber anderweitig gut gekannten Magenschleimhäuten anderer Thiere

1) Vergleiche hierzu: Vorlesungen über vergleichende Anatomie von G. Cuvier (Meckel's Uebers.) III. Theil 1810, pap. 391. - Medicinische Zoologie von J. F. Brandt und Ratzeburg, I. Bd. 1829, pag. 19. Dort ist auch die ältere Literatur citirt. - Lehrbuch der vergleichenden Anatomie der Wirbelthiere von Siebold und H. Stannius 1846. II. Theil. pag. 425. - Leydig: Lehrbuch der Histologie 1857, p. 315. 
lehrten, dass, wie in dem vorliegenden Bibermagen, nach einiger Zeit in diluirtem Alcohol Oberflächenepithel und Pylorusdrüsenzellen nicht mehr aufzufinden sind; während in den Labdriusen Zellen erhalten bleiben.

$\mathrm{Ob}$ in den Driisen des Fundus und der grossen Curvatur, ob in der grossen zusammengesetzten Drïse Haupt- und Belegzellen vorkommen, liess sich nicht entscheiden. Doch sollte man erwarten, dass bei der anscheinenden Einfachheit, die Drüse aus dem Connex des Bibermagens operativ zu entfernen, an diesem Object eine elegante Lösung der Frage nach der Pepsin- und Säurebildung im Säugethiermagen gewonnen werden könnte.

\section{Der Magen der Vögel.}

In der Absicht auf vergleichend anatomischem Wege Beweise für die Pepsinbildung in den Belegzellen der Säugethiere $z u$ erbringen, war in der ersten dieser Mittheilungen Einiges tiber den Bau der Labdrüsen der Vögel erwähnt worden. Seit jener Zeit hat Ed. Remouchamps ${ }^{1}$ ) eine Beschreibung der Magendrüse von Rhea americana geliefert. Ueber den feineren Bau der Drüsenzellen sind keine Angaben gemacht; doch interessirt die Notiz, dass nur eine Zellenart in den Schläuchen sich finde.

Bei eignen erneuten Untersuchungen war die Aufmerksamkeit in Wesentlichen auf die Coustatirung einer dem Bibermagen analogen Vertheilung der Druisenformen und die Auffindung von Hauptund Belegzellen gerichtet. Das Folgende wird zeigen, wie weit die gèhegten Erwartungen gerechtfertigt waren.

Bei einem Haushuhn (Gallus domesticus) maass der ganze Drtisenmagen im Längendurchmesser $4,5 \mathrm{~cm}$. Davon kamen $3 \mathrm{~cm}$ auf die Zone der etwa 50 bis 60 flaschenförmigen zusammengesetzten Labdrïsen, deren Dicke in der Mitte dieser Zone am mächtigsten ist (etwa $5 \mathrm{~mm}$ ) und nach beiden Richtungen, nach oben und unten hin, gleichmässig abnimmt. Auf diesen Abschnitt folgt ein zweiter $1,5 \mathrm{~cm}$ langer, mit einfach schlauchförmigen, kaum $1 \mathrm{~mm}$ hohen Labdrüsen. Der Muskelmagen mit hornigem Belag macht den Beschluss.

1) Ed. Remouchamps: Sur la glande gastrique du Nandou d'Amérique. Archives de Biologie, publiées par Ed. van Beneden et $\mathrm{Ch}$. van Ba mbeke. Vol. I, p. 583, 
Dass der Muskelmagen der Vögel dem Pylorustheil des Magens anderer Wirbelthiere homolog sei, hat Retzius überzeugend dargethan. Dies lässt sich auch bei Raubvögeln, denen ein horniger Muskelmagen fehlt, leicht erkennen. Untersucht man den Magen von Raubvögeln, so findet sich auch hier die Dreitheilung wieder. Zuerst ein Abschnitt mit grossen flaschenförmigen Drüsensäcken; darauf einfache Labdrüsenschläuche und schliesslich der Pylorustheil. Mir sind die Magenschleimbäute vom Mänsebussard (Falco buteo) und von der Schleiereule (Strix flammea) bekannt geworden. Als Paradigma sei der Magen von Strix flammea etwas eingehender beschrieben. - Das untersuchte Thier batte einen Tag gehungert.

Der Oesophagus ist mit dicht gestellten Schleimdrüsen ausgestattet. Der erste Magenabschnitt trägt die ca. $3 \mathrm{~mm}$ mächtige Schicht zusammengesetzter flaschenförmiger Labdrïsen. Bekanntlich sind in jeder Drüse eine grosse Anzahl von Drüsenschläuchen enthalten, deren Secret in einen gemeinschaftlichen, central gelegenen Ausfiihrungsgang entleert wird. Zwischen den Mündungen der weitbauchigen und schmalhalsigen Drüsencomplexe zieht die Schleimdrüsenschicht des Oesophagus continuirlich weiter, so dass sich ein Bild präsentirt, wie es Bergmann ${ }^{1}$ ) bereits vom Staar dargestellt hat ${ }^{2}$ ). Die einzelnen Tubuli der grossen flaschenförmigen Labdrisen tragen nur eine Art granulirter Zellen. Die Granula werden in Ueberosmiumsäure gebräunt.

Auf diese erste, nur von einer dünnen Muskelhülle umkleidete Zone der Magenschleimbaut folgt ein stark muskulöser Theil mit einfachen scblauchförmigen Drüsen besetzt. Die Architektonik der Zellen erinnert an die des Pankreas: einwärts rom Kern eine in Ueberosmiumsäure sich bräunende fein granulirte Zone; der Membrana propria zugewandt ein heller protoplasmatischer Abschnitt.

Was die Deutung der Driisen in diesen beiden $A$ btheilungen der Magenschleimhaut anlangt, so wird man in Grundlage der Form der Schläuche sich dafür entscheiden, die zusammengesctzten Drüsen mit den Oesophagealdrüsen der Frösche und die einfachen mit den sogenannten Labdrüsen der Frösche zu vergleichen. Dafür spricht auch die

1) Müller's Archiv 1862, Taf. XIV A, Fig. 1.

2) Die Lage der Brunner'schen Drüsen unter der eigentlichen Schleimhaut des Duodenum hat viel Aehnlichkeit mit dem hier geschilderten Verhalten. Ebenso liegen die Oesophagealdrüsen der Frösche tief unter dem Epithel der Oberfläche. 
Reihenfolge der Drüsen und die relative Grösse der in den Zellen enthaltenen Granula. Die Granula sind in den Oesophagealdrüsen und in den zusammengesetzten Drüsen des Vogelmagens grösser als in den Labdrisen der Frösche und in den Schläuchen der soeben beschriebenen zweiten Abtheilung des Drüsenmagens der Vögel. Trotzdem ist man vorläufig noch nicht völlig berechtigt, die Drüsenzellen in den zusammengesetzten Drüsen des Vogelmagens für Hauptzellen und die der einfachen Schläuche für Belegzellen zu erklären, da, wie es scheint, die Anordnung der Granula in den Zellen gerade die umgekehrte ist.

Ein eigentlicher Muskelmagen, d. h. ein von quergestreiftel Husculatur umhüllter, innen mit erhärtetem Drüsensecret ausgekleideter Pylorustheil fehlt. Auf den eben beschriebenen zweiten Abschnitt folgt aber noch ein dritter von dem Baue der Pyloruszone der übrigen Wirbelthierklassen. Die Drüsenschläuche werden gegen das Duodenum zu immer niedriger. Osminmsäure bräunt sie ebensowenig als die Schleimdrüsen des Oesophagus.

\section{Dep Vorderdarm der Reptilien ${ }^{1}$. \\ Figg. 10, 14, 15, 16, 17, 18, 19.}

Mit Bezug auf die eigenartige Vertheilung der Labdruisen im Vorderdarm der nächsten Wirbelthierklasse, der Amphibien, musste anch der Oesophagus der Reptilien eingehender durchmustert werwerden. Auch bei den Reptilien herrschen die grössten Verschiedenheiten im Vorkommen von Oesophagealdrüsen. Dass der Oesophagus von Lacerta agilis und von Coluber natrix drïsenlos sei, dass dagegen bei Testudo graeca eine schon mit freiem Ange erkennbare Drüsenschicht im Oesophagus sich finde, hat bereits Leydig angegeben. Unterschiede im Baue der vorderen und weiter gegen den Pylorus gelegenen Labdrïsen von Coluber natrix hat Langley und das Vorkommen eines ächten Pylorustheiles Edinger beschrieben. Meine eigenen Untersuchungen bezieben

1) Vergleiche hierzu: L eydig: Anatomisch-histologische Untersuchungen über Fische und Reptilien 1853. - Histologie 1857. p. 312. - Partsch: Archiv für mikroskopische Anatomie Band XIV. - Edinger: Dasselbe Archiv, Bd. XVII. - Machate: Zeitschrift für wissenschaftliche Zoologie, Bd. 32. - Langley: Philosophical Transactions of theRoyal Society. Part. III. 1881. 
sich auf Lacerta agilis, Anguis fragilis, Emys europaea, Testudo graeca, Coronella laevis.

Lacerta agilis. Der Oesophagus ist bis auf einen kleinen, etwa $2 \mathrm{~mm}$ langen, an den Magen anstossenden Ring frei von Drüsen. Das Oberflächenepithel, auf breiten confluirenden Längsleisten arrangirt, besteht aus Flimmerzellen und Becherzellen mit breiter Theca und feinem, kreisförmigem Stoma.

Der Oesophagus ist durch eine circuläre, am anfgeblähten Präparat nach innen vorspringende Falte gegen den Magen abgesetzt. Ueber diesen Ring hinaus gehen die im unteren Bezirk des Oesophagus beginnenden Druisen weiter in den Magen hinein. Das Oberflächenepithel verändert seinen Character schon im Oesophagus von der Stelle an, wo die Drüsen beginnen und setzt sich in derselben Form in den Magen hinein fort. Dies ist ein wesentlicher Unterschied gegenüber dem Verhalten der Oberflächenepithelien beim Frosch, wo die erste, pepsinbereitende Drïsenart des Vorderdarms im Oesophagus unter einer Decke von Becher- und Wimperzellen liegt. Bei Lacerta hört jedoch in der Region der beginnenden Drüsenbildung im Oesophagus jede Wimperung auf und man findet nur Schleimzellen, deren Secret bei verschiedenen Präparationsmethoden ausgepresst wird, so dass eine Zelle mit Kern, Protoplasmafuss und hohlem oḅeren Aufsatz zurückbleibt. In Ueberosmiumsäure bleiben die Zellen unverändert; der Schleim wird nicht eliminirt. Die Höhe der schleimigen Zone variirt sehr. Zuweilen erreichen die nach abwärts zugespitzten Zellen eine enorme Länge; in einem Falle mass der protoplasmatische Fuss $36 \mu$, die schleimführende Theca $18 \mu$. In diesen Zellen ist kein kreisförmiges Stoma wie bei den Becherzellen vorgebildet; auch besitzen die Zellen keine Membran ${ }^{1}$ ). Die Drüsen selbst sind mehrbuchtige

1) Man würde in der Histologie des Thierreichs jede Differenz in der Darstellung vermeiden können, wenn man consequent nur das eine ,M e mb r a n" nennen würde, was aus Zellen besteht, wie die Membrana granulosa bei den Eiern der Wirbelthierc oder die Cysten- und Follikelhaut der Samenzellenkugein.

Sobald die thierische Zelle ibre amoeboide Beweglichkeit aufgegeben hat, erhärtet ihre Rinde mehr oder weniger und gibt dadurch der Zelle eine definitive Gestaltung, ohne dass Zellleib und Rinde chemisch different zu sein brauchen.

In anderen Fällen umgeben sich die Zellen allseitig, so die Eier 
Schläuche, zwischen deren einzelne Zipfel, wie bei den zusammengesetzten Labdrüsen der Vögel, Blutgefässcapillaren eindringen. Das Innere der Zellen ist mit grossen, in Osmiumsäure sich bräunenden Granulis gefüllt; die Vermehrung derselben schreitet wie bei den Oesophagealdrisen des Frosches und den pancreatischen Drüsen aller Wirbelthierklassen von dem Centrum - $d . h$. von dem am Lumen anstossenden Theile der Zelle - gegen die Peripherie vor. Haben Eidechsen längere Zeit gehungert, so sind die Drisen verkleinert; nur in wenigen Zellen sind vereinzelte Granula erhalten. Fuittert man ein Thier nach längerem Fasten, so füllen sich die Zellen in den ersten Stunden der Verdauung wieder mit den groben Körnern; nicht selten findet man in den Schläuchen Zellen, die wie leer aussehen, keine Granula enthalten und deren Kern von derselben Beschaffenheit ist, wie Kerne in pancreatischen Zellen des Landsalamanders, die abgestorben sind und eben ausgestossen werden.

Auf diese erste Art von Magendrüsen folgt, in der Mitte des Magens an Grösse zunehmend, eine zweite Form von Drüsen, die zu mehreren vereinigt in runden, schlitz-oder T-förmigen Griibchen

der meisten Wirbellosen, mit einer „Ḧ̈ lle“, wie dic Pflanzenzelle mit einer Celluloseschicht. An vielen Oberflächen, in den Buchten und Binnenrïumen epithelialer Bildungen wird einseitig entweder eine continuirliche oder in den Zellgrenzen unterbrochene, erhärtete, oft von feinen Poren durchsetzte Hülle abgeschieden. Diese Hüllen sind wie die Cellulose vom ursprünglichen Zellprotoplasma verschieden und gehören vielleicht alle wie die Cellulose zu den Kohlehydraten.

Die Becherzellen haben um den schleimigen Inhalt eine isolirbare Hülle; ob diese nur die erhärtete Rindenschicht des ursprünglichen protoplasmatischen Kopfes der Zelle sei, oder, ob sie einem complicirten Chemismus ihren Ursprung verdankt, wie eine Cuticula, muss vorläufig unentschieden bleiben. Sie ist etwas für sich Bestehendes, aber zugleich mit ihrem Inhalt aus der anfangs protoplasmatischen Zelle hervorgegangen.

Die Hülle der Becherzellen ist an der freien Fläche kreisförmig durchbrochen; bei den Schleimzellen der Magenoberfläche ist diese Oeffnung meist sechseckig, weil hier gleichartige Zellen aneinandergelagert sind und die gleichzeitige Dehnung aller Zellen jeder einzelnen einen gewöhnlich sechsseitigen Querschnitt gibt. Sind nicht alle Zellen gleichzeitig mit Schleim gefüllt, also mit weichen protoplasmatischen, nackten oder bewimperten Zellen gemischt, so kommt die Becherform der Schleimzellen zu Stande, aus der sich durch ideelle Verlängerung der Mündung die einzellige Drüse mit langem Ausführungsgang ableiten lässt. 
münden. Die Grübchen sind am frischen Präparat 0,5-1,5 mm von einander entfernt. Diese Drüsen sind gestreckt schlauchförmig. Das Epithel der Oberfläche ragt verschieden weit in den Hals der Schläuche hinein. Im eigentlichen Drüsenkörper sind zwei Zellarten vorhanden. Die eine Art ist namentlich während der Verdauung mit feinen senkrecht zum Lumen der Drüse in Reihen geordneten Körnchen und Stäbchen gefiillt. Diese durch Osmiumsäure braun färbbaren Körnchen umgeben einen grossen, hellen runden oder ovalen Kern. Die andere Zellart bildet Schleim und kann weder durch Osmiumsäure noch durch Carmin gefärbt werden. In Beale'scher Carminlösung und beim nachherigen Auswässern des Präparats geht der schleimige Inhalt der Zellen verloren, und diese zeigen ein ähnliches Aussehen, wie die Epithelien der Magenoberfläche, bei gleicher Behandlung. Die erhärtete Rindenschicht der Zellköpfe ragt als leere Hiulle über den protoplasmatischen kerntragenden Fuss der Zelle hinaus: der Schleim ist durch Einwirkung des Wassers gequollen und nach Aussen entleert worden.

Im Alkohol geht die feinere Structur aller Zellen zu Grunde. Gegen den Pylorus zu wird die Muskulatur des Magens mächtiger, die Driisenschicht viedriger. Die Schlïuche sind einfach oder verästigt und enthalten Schleimzellen, die in Osmiumsäure nicht gebräunt werden.

Anguis fragilis bat einen lang gestreckten Vorderdarm. Der Oesophagus flimmert nicht. Das Epithel der Oberfläche besteht ans langgestreckten keilförmigen Zellen mit protoplasmatischem Fuss und einem kleinen sechsseitigen Aufsatz mit schleimigem Inhalt. Vereinzelt und durch grössere Zwischenräume getrennt sind der Schleimhaut flache Schleimdrïschen eingelagert. Beim Uebergang des Oesophagus in den Magen beginnen die Labdrüsen, die durch Osmiumsäure je nach dem Grade ihrer Füllung mit körnigem Inhalt mehr oder weniger lebhaft gebräunt werden, während die Oesophagealdriisen und die Drüsenschläuche der Pylorusregion sich indifferent gegen die Ueberosmiumsäure verhalten. Die Labdriisenzone zerfällt wie bei Lacerta in zwei Abschnitte; ein vorderer mit grossen Granulis in den Zellen der niedrigen, buchtigen Drüsen; ein hinterer Abschnitt mit vorwiegend fein gestrichelten Zellen, zwischen denen auch einige Schleimzellen sich finden. Die Strichelung der Zellen ist auch hier auf die radiäre, reihenweise Anordnung feiner Körnchen und Stäbchen zurückzuführen. Im Vergleich 
zu Lacerta sind alle in Betracht kommenden Verhältnisse bei Anguis zarter und kleiner, so dass der Vorderdarm von Anguis nur die Schleimdrüsen und der von Lacerta das Wimperepithel im Oesophagus als specifisches Merkmal aufweist. Im Uebrigen ist der Bauplan und seine detaillirte Durchflihrung bei beiden Species nahezu derselbe.

Während aber bei der Eidechse und der Blindschleiche im eigentlichen Labdrüsenabschnitt des Magens zwei verschiedene Drüsenarten mit typischen Zellformen aufeinanderfolgen, ist im Magen der Schildkröten und der von mir untersuchten Coronella laevis eine andere Anordnung vorhanden; es fehlen die Pepsindrüsen mit den grossen Granula in den Zellen, welche bei Rana im Oesophagus und bei Lacerta und Anguis im vorderen Abschnitt des Magens sich finden. Dafür sind zwischen den Schläuchen der Labdrüsenzone der Schildkröten mit dem Charakter der Labllüisenschläuche des zweiten Magenabschnittes von Lacerta und Anguis oder des ersten Magenabschnittes von Rana, andere Schläuche eingeschaltet, die während des Hungers ganz das Aussehen von Schleimdrtisen zeigen. Ebenso bäufig sind aber auch in demselben Schlauch beide Zellenarten in der Weise gemischt, dass, wie Machate dies von Emys europaea hervorgehoben hat, kurze Strecken desselben Schlauches von den grösseren fein granulirten Zellen austapezirt, anderc Strecken wieder mit hellen, schmalen cylindrischen Zellen besetzt sind. $\mathrm{Ob}$ die hellen Zellen Hauptzellen oder wirkliche Schleimzellen seien, habe ich nicht entschejden können, da zu gewissen Zeiten beide dasselbe Aussehen haben können. Zerzuptungspräiparate lassen sich nicht mit gentigender Klarheit herstellen. Feine Schnitte durch die Schleirnhaut des eben getödteten Thieres zeigen nicht mehr als Osmiumsäurepräparate und, abgesehen von der eintretenden Bräunung der granulirten Zellen, dasselbe Bild. In absolutem Alkohol wird zwar das Aussehen gegen den frischen $\mathrm{Zu}$ stand wesentlich verändert, aber die Unterschiede der beiden Zellenarten treten recht deutlich hervor. Da mir aber nur Thiere zu Gebote standen, die lange gehungert hatten und es nicht gelingen wollte, dieselben zum Fressen zu bringen, so ist es möglich, dass die bellen Zellen zu anderen Zeiten doch jene bei allen Wirbelthieren wiederkehrenden grossen Granula bilden. In meinen Präparaten waren sie gewiss nicht vorhanden. Es müssen also fortgesetzte Untersuchungen diesen Punkt weiter aufklären. - Die 
aufgelöste Labdrüsenzone der Schleimhaut wirkte peptisch. Da nun bei den Schildkröten in den Labdrüsenschläuchen sich Zellen finden von dem Charakter der Belegzellen der Säugethiere und den Zellen im ersten Abschnitt des Froschmagens - von letzteren hat Langley noch kürzlich erst Fermentbildung nachgewiesen so wird man die peptische Wirksamkeit der Labdrüsenschleimbaut des Schildkrötenmagens diesen fein granulirten, in Osmiumsäure sich bräunenden Zellen zuschreiben. Dies um so eher, als es überhaupt fraglich ist, ob bei den Schildkröten Hauptzellen vorkommen.

Der von Machate gegebenen Beschreibung des Vorderdarmes von Emys europaea wäre kaum etwas hinzuzufügen; nur habe ich häufig gesehen, wie die hellen Schläuche noch eine Strecke weit in die Muscularis mucosae hineinragten, ein Verhalten, wie es bei anderen Wirbelthieren wohl nicht bekannt sein dürte. Beim Uebergang des Oesophagus in den Magen finden sich einige Schleimdruisen, die sich an Präparaten aus absolutem Alkohol schon makroskopisch durch ihre grössere Resistenz und hellweisse Färbung von der tibrigen weicheren und etwas grau gefärbten Schleimhaut unterscheiden.

Testudo graeca. Die Länge des Magens einer nach dreimonatlichem Winterschlaf getödteten Testudo graeca betrug $8 \mathrm{~cm}$, wovon $3,3 \mathrm{~cm}$ auf den grauröthlichen Anfangstheil, das übrige auf den bis zum Pylorus hinziehenden weisslichen Abschnitt kamen.

Der Oesophagus enthält grosse nah gestellte Schleimdrüsen, die beim Aufspannen des aufgeschnittenen Vorderdarms als opake Körner mit blossem Auge in der Schleimhaut sichtbar sind. Um diese Zeit waren die Drüsen prall mit Schleim gefullt.

Der geröthete Theil der Magenschleimhaut hat zweierlei Schläuche, wie bei Emys. Die granulirten in Osmiumsäure gebräunten Zellen haben ein fein radiär gestricheltes Aussehen von der regelmässigen Anordnung der kleinen Körnchen; das Protoplasma der Zellen am Lumen der Schläuche ist frei von Einlagerungen, homogen. Wo der belle Theil der Magenschleimhaut beginnt, werden diese Belegzellen immer seltener; die Zahl und die Länge der. Schläuche nimmt bis zum Pylorus ab, und bald sind alle Schläuche von Schleimzellen erftillt, die in diesem Stadium wie im Oesophagus viel schleimige Masse enthalten.

Die Schläuche der gerötheten Schleimhantzone, welche ganz mit den hellen Zellen ausgekleidet sind, sind breiter als die ganz oder theilweise mit Belegzellen erfullten. In den hellen Zellen habe ich 
ebensowenig, wie bei Emys Granula aufgefunden, weder frisch, noch nach Erhärtung der Schleimhaut in Ueberosmiumsäure.

Coronella laevis. Der Oesophagus ist drüsenlos, trägt ein aus Becher- and Flimmerzellen bestehendes Epithel. Der vordere Magenabschnitt hat zusammengesetzte, der folgende Theil lange einfache Schläuche; beide jedoch mit derselben Zellenart belegt, in denen gleichartige grosse Granula angehäuft sind. Im Hals der Drüsen finden sich viele helle in Ueberosmiumsäure nicht gefärbte Zellen. Der Pylorustheil mit seinen Schleimdrüsen macht den Beschluss.

\section{Der Vorderdarm der Amphibien ${ }^{1}$ ). \\ Figg. 13, 32, 33, 34.}

Genauer untersucht sind bis jetzt Rana temporaria und esenlenta, Bufo variabilis, Triton taeniatus und Triton cristatus. Der Beschreibung von Langley wird man kaum Etwas hinzuzufugen haben. Ueber die von ihm gewählte Nomenclatur ist schon oben die Rede gewesen. Von mir wurden noch weiter untersucht Salamandra maculosa und Triton helveticus; der Vorderdarm ist im Bau ubereinstimmend mit dem des von Langley geschilderten Triton taeniatus und cristatus. Demgemäss kommen den Amphibien drei Drüsenarten im Vorderdarm zu; da Schleimdrüsen im Oesophagus allgemein zu fehlen scheinen, und nur ein auf Kämmen und Riffen angeordnetes Epithel von Wimper- und Becherzellen im Oesophagus sicb findet. Die erste Drisenart, aus Hauptzellen aufgebaut, liegt entweder im Oesophagus (Rana) oder am Uebergange des Oesophagus in den Magen (Bufo) oder wie bei Tritou und Salamandra im Anfangstheil des Magens. Die zweite Drisenart besteht aus einfachen Schläucben, die mit Belegzellen ausgekleidet sind. Es ist seit $\mathrm{Heiden} \mathrm{n}$ ain bekannt, dass zwischen den Belegzellen sich auch hier und da Schleimzellen vorfinden. Die Schleimdrüsen des Pylorustheiles bilden die dritte Drüsenart des Vorderdarmes der Amphibien.

Vom Frosch ist nachgewiesen, dass die zusammengesetzten Drtisen des Oesophagus Pepsin in alkalischer Lösnng, und dass

1) Vergl. hierzu: R. Heidenhain, dies Archiv Bd. VI, p. 394. - C. Partsch, d. Arch. Bd. XIV, p. 179. - N. Langley, Philosoph. Transactions of the Royal Suc. III. Part. 1881. 
die Belegzellen eine saure Pepsinlösung absondern. Langle ${ }^{1}$ ) hat durch vergleichende Verdauungsversuche bei Triton taeniatus den Nachweis erbracht, dass die Zone der Belegzellen mehr Pepsin enthalte, als die der Hauptzellen. Dies ist zwar bei andern Thieren nicht der Fall. Doch wird durch Langley's Beobachtung die Pepsinbildung in den Belegzellen a fortiori bewiesen.

Es braucht wohl nicht besonders hervorgehoben $\mathrm{zu}$ werden, dass die mit Haupt- und Belegzellen benannten Zellen des Amphibiendarmes in allen Punkten: der Grösse und der Art der Aufspeicherung, der Löslichkeitsverhältnisse und dem Verhalten ihrer Granula gegen Ueberosmiumsäure mit den gleichnamigen Gebilden der Säugethiere völlig übereinstimmen. Die Maasse sind natürlich nicht dieselben, wohl aber alle bei Säugethieren hervorgebobenen relativen Beziehungen.

\section{Der Magen der Fisclie ${ }^{2}$ ).}

Taf. XVII, Fig. 29.

Die Classe der Fische zeigt die mannigfachsten Variationen im Bau der Schleimbaut des Vorderdarmes und in engem Zusammenhang damit auch des Pancreas. Vielen Fischen geht ein peptisches Ferment gänzlich $a b$ und man sucht vergebens die Drüsen, welche nach dem Typus der Labdrusen gebaut wären. Das Pancreas ist bei diesen Arten, wie Legou is ${ }^{3}$ ) im Anschluss an die Entdeckung We ber's gezeigt hat, disseminirt und die einzige eiweissverdauende Drise des Vorderdarmes. Was die Behauptung Krukenberg's anlangt, es fehle manchen Teleostiern ein Pancreas, so kann ich nach meinen Untersuchungen wenigstens für den Hecht nicht zustimmen. Alessandrini ${ }^{5}$ ) hat beim Hecht ein Pancreas beschrieben, und es kommt diese Drtise in der That

1) Langley: Philosoph. Transactions Part III. 1881, p. 690.

2) Vergleiche hierza: Edinger: Ueber die Schleimbaut des Fischdarmes. D. Archiv Bd. XIII und die dort angeführte Literatur. - Krakenberg: Untersuchungen aus dem physiolngischen Institute der Universität Heidelberg. I. Bd. Heft 4, II. Bd. Heft 1 und 4.

3) Legouis: Annales des sciences naturelles. 1873, V. série (Zoologie), T. 17 et 18. (Recherches sur les tubes de Weber et sur le pancréas des poissons osseux.)

4) Weber: Ueber die Leber von Cyprinus carpio in Meckel's Arch.1827.

5) Alessandrini: Nov. Comment. Acad. Bonon. 1835, T. II. 
beim Hecht vor. Sie liegt in einer grossen Fettanbäufung verborgen. Die Schlänche der Drüse sind zur Zeit der Verdaunng mit den charakteristischen Granula gefültt; es lässt sich ein tryptisches Extract daraus gewinnen, während die gleichartig behandelte Leber keine verdauende Wirkung zeigt ${ }^{1}$ ).

Bei den Cyprinoiden (Cyprinus carpio) geht der Oesophagus

1) Zur bistorischen Entwicklung der Kenntnisse vom Pancreas der Fische:

Cuvier et Valenciennes (Histoire nat. des poissons, Paris 1828 p. 503, T. I): Labrus, Silurus, Cyprinus, Esox fehlt das Pancreas und das System der Appendices pyloricae.

Weber (Meckel's Archiv 1827, pag. 298) entdeckt bei Cyprinus die nach ihm benannten Gänge und spricht die Vermuthung aus, dass die Leber bei den Cyprinoiden zugleich die Function eines Pancreas habe.

Allessandrini (Nov. Comment. Acad. Bonon. 1835, T. II) weist bei Esox lucius und Accipenser sturio ein Pancreas nach, soweit dies mit Hülfe der ausschliesslich anatomischen Methoden möglich ist. Alessandrini glaubt allen Fischen ein gesondertes Pancreas zusprechen zu müssen und zieht Weber's oben angeführte Meinung und die Cuvier's, dass bei den Fischen mit mangelndem Pancreas und fehlenden Appendices pyloricae der Dünndarm drüsenreicher zu sein pflege, in Zweifel.

Joh. Müller (Müller's Arch. 1840, pag. 132 Anmerkung) beschreibt bei Lota das Vorkommen von Appendices pyloricae und eines auch mikroskopisch von ihm untersuchten Pancreas. Ebenso kommt nach Müller dem Wels, Aal und Hecht ein drüsiges Pankreas zu, während diesen Fischen die Pylorialanhänge fehlen. (Die Bedeutung der Appendices pyloricae als Ausstülpungen der Darmwand, "von demselben Baue wie diese" hat $\mathrm{E} d$ in $\mathrm{g}$ or nachgewiesen. D. Arch. Bd. XIII).

Stannius gibt 1846 in Müller's Archiv eine Zusammenstellung seiner in Brockmann's Dissertation niedergelegten Entdeckungen über das Pancreas der Fische.

Aus der oben angeführten grossen und umfassenden Arbeit des Pater L egouis sei noch erwähnt, dass von diesem Forscher (1. c. T. 17, pag. 41) Esox lucius, Muraena, Conger, Anguilla, Pleuronectes, Accipenser sturio ein Pancreas zugeschrieben wird.

Krukenberg weist mit Hülfe von Verdauungsversuchen die Fermente der pancreatischen Drüsen nach. - Nur sei die Bemerkung gestattet, dass das Hepatopancreas der Fische keine einheitliche Drüse darstellt wie bei Wirbellosen; die weissliche Pankreassubstanz ist vielmehr von der gelblichen oder bräunlichen Lebermasse völlig histologisch getrennt. Die Drüsen sind nur makroskopisch innig gemischt. Auszüge der frei präparirten Knötchen des Pancreas von Cyprinus carpio wirken pancreatisch; die Auszüge der isolirten Lebersubstanz verdauen kein Eiweiss. 
in einen erweiterten Abschnitt über, der dem Bau und der Verrichtung seiner Schleimhaut gemäss als Duodenum anzusprechen ist; an der Grenze beider Abschnitte miinden die Ausführungsgänge der Leber und des disseminirten Pancreas. Bei den Petromyzonten fehlen ebenfalls die Pepsindriisen; es kommen aber zwei Drüsen vor, von denen man vermuthen darf, dass sie fermentirende Secrete liefern. Die erste Drüse liegt am Uebergange des Oesophagus in den Darm, die zweite in der Substanz der Leber; beide sind von einerlei Bau. Sie bestehen aus Schlänchen, die sich an den zur Untersuchung bis jetzt verbrauchten Thieren nur zum kleineren Theil in Ueberosmiumsäure bräunten. Auch fehlten in den Zellen die charakteristischen Granula, die im Pancreas aller Wirbelthiere zu finden sind. Ob demgemäss die Drüsen ein Pancreas seien, kann vorläufig nicht entschieden werden, da das zu Gebote stehende Material zu gering war. Selbst wenn es ausreichte, so wtirde es doch wenig Erfolg versprechen, da die Meeresneunaugen (Petromyzon marinus), bevor sie im Rhein gefangen werden, sicher längere Zeit schon geliungert haben. Wenigstens war bei den von mir untersuchten Exemplaren der Darm absolut leer. Es wiurde aber interessant sein, die Bedeutung der Driisen mit Sicherheit zu kennen, weil bei Cyprinus nur ein disseminirtes Pancreas vorkommt, und bei vielen anderen Wirbelthieren die vorderen Pepsindrüsen mit ihren grossen Körnern, der Entwicklung derselben in den Zellen und der zusammengesetzten Bauart, sehr an das Pancreas erinnern und, wie beim Frosch, auch im Oesophagus gelegen sein können. Man könnte desshalb vermuthen, die Drüsen am Uebergang des Oesophagus in den wimpernden Darm der Petromyzonten entsprächen der ersten, ausschliesslich Hauptzellen enthaltenden Form von Labdrisen bei den iibrigen Wirbelthieren. Die nicht weit abwärts in. der Lebersubstanz befindliche und auf Längsschnitten durch die Mitte der am Darm befestigten Leber sichtbare weissliche Druise würde dann das compacte Pancreas der anderen Wirbelthiere darstellen. Die Drüsen am Uebergange des Oesophagus in den Darm waren Bojanus bekannt; Langerhans hat sie beim Querder entdeckt; Stannius gibt über die in der Leber gelegene Drïse eine kurze Notiz.

Der Vorderdarm der höher stehenden Fische hat, wie am Hecht leicht gezeigt werden kann, schon die Organisation der folgenden Klassen; im eigentlichen Magen kommen drei Drüsenarten 
vor. Schleimdrüsen fehlen im Oesophagus, dessen schleimproducirendes Epithel auf mannigfach gezackten Kämmen in mehrfachen Lagen angeordnet ist. Im vorderen Abschnitt des Magens findet man Labdrüsen mit groben Körnern in den gleichartigen Zellen. In Ueberosmiumsäure brännen sich die Körner; die Kerne der Zellen treten nicht deutlich hervor. Die, Zellen selbst sind den Hauptzellen vergleichbar. Auf diese Zone folgt eine zweite, mit Drïsenschläuchen besetzt, deren Hals relativ lang und deren Drüsenkörper recht kurz ist. Die Zellen sind fein granulirt; die Granula radiär zum Lumen der Schläuche geordnet, wie dies von den Belegzellen der Reptilien und Amphibien oben berichtet wurde. : In Ueberosmiumsäure werden die Granula gebräunt und die Kerne treten deutlich hervor. Auf diese Schleimhautzone folgt der kurze, an einem zweipfundigen Hecht kaum $1 \mathrm{~cm}$ messende Pylorustheil mit zusammengesetzten kurzen Schleimdrisen, die sich in Ueberosmiumsäure nicht bräunen.

Es wäre einer erneuten Untersuchung vielleicht nicht unwerth, ob bei den Fischen mit peptischem Magensaft immer zwei Formen von Pepsindrüsen sich finden, und ob die erste Art derselben ein alkalisches, die zweite Art ein saures Secret liefere. Der Nachweis des letzteren Verhaltens fehlt ibbrigens auch noch für Vögel und Reptilien, während er beim Frosch bereits erbracht ist.

\section{Abschnitt.}

Von der Regeneration der Drüsenzellen und den Beziehungen dieses Vorganges zur Secretion.

Figg. 25, 27, 28, 30, 31, 36, 37, 44.

Es möchte heutzutage ${ }^{1}$ ) wohl nur noch Wenige geben, die das Wesen der Secretion in einer Ausstossung von Zellen finden wollen. Man ist daruber einig, dass nicht weiterhin, wie es früher vielfach beliebt wurde, Hoden und Eierstock den 'Typus einer secernirenden Drüse abgeben können. Die Eliminirung der Geschlechtsproducte hat mit einem Secretionsvorgange nichts gemein. Samen

1) Vergl. hierzu: Pflüger: Die Endigungen der Absonderungsnerven in den Speicheldrüsen. Bonn 1866, p. 41-45. 
und Ei bethätigen nur lebend ihre Functionen; sie werden desshalb lebend und als modificirte Zellen ausgestossen. Die Secrete können unbeschadet ihrer Wirksamkeit mit den dem Leben feindlichsten Reagentien behandelt werden; sie sind nur die ausgestossenen Producte lebender Zellen.

Wie es Beale zuerst gethan hat, soll man die Secretbildung mit der Entwicklung der höheren Gewebe vergleichen. In der That gibt es alle Uebergänge von dem einen Typus zum anderen. Man könnte wohl gegen den intendirten Vergleich den Einwand machen, dass die Secrete, ob fest oder flussig, immerhin etwas schnell Vergängliches; die höheren Gewebe dagegen, wie die quergestreifte Muskulatur, sehr stabiler Natur seien. Doch gibt es auch bleibende Secrete. Wälırend die Zellen des Clitellum vieler Würmer die anfangs flüssige und erst als Coconhülle zu einer chitinigen Haut erstarrende Masse absondern, die dem elterlichen Organismus demgemäss als Secret entzogen wird, liefern für den Leib des Thieres die sogenanuten Matrixzcllen der Cuticula dasselbe Secret in bleibender Form. Die Zelleu bleiben sogar, wie die Zellenreste der quergestreiften Muskulatur, innig mit der Cuticula verbunden. Die Pylnrusschleimhaut der meisten Wirbelthiere producirt ein tlüssiges Secret, das schnell eliminirt wird; im Pylorustheil der körnerfressenden Vögel - dem Muskelmagen - wird ein bleibendes Secret abgesondert, das zu einer dicken, starren Reibfläche erhärtet.

Wie wir nun aber seit langer Zeit schon gewohnt sind, die Muskelzellen als etwas Stabiles zu betrachten und die Fähigkeit der Regeneration zu Grunde gegangener quergestreifter Substanz. der verkïmmerten ursprïnglichen Bildungszelle zuzuschreiben, so nehmen wir ein Gleiches für viele secretorische Zellen an. Somit wïrde die Entwicklung der Secrete und Gewebe nach einem gemeinsamen Schema ablaufen und ein durchgreifender Unterschied erst in der Function gegeben sein: es käme den Drüsenzellen die Chemie, den Gewebezellen die Mechanik des lebenden Haushaltes zu. Diese Unterscheidung schliesst nicht aus, dass die das Leben charakterisirenden chemischen Vorgänge auch in den Gewebezellen sich abspielen und Secrete gelegentlich als passive mechanische Vorrichtungen dienen. Man wïrde demnach histologisch die ursprüngliche Muskelzelle der secretfreien Drüsenzelle, beziehungsweise dem der Membrana propria zugewandten Abschnitt der- 
selben, das Secret oder die dem Lumen zugewandte Zone des Secretionsmaterials der quergestreiften Substanz vergleichen, und nur dem Protoplasma und dem Kern beider Zellarten eine grössere Lebensdauer und zugleich dic Fähigkeit der Regeneration znsprechen. Bei alledem dürfte es nicht überflüssig erscheinen, für diese Ansicht einen sicheren und unumstösslichen Beweis zu haben. Die Wandlungen im Aussehen der Zellen im Laufe einer Secretionsphase sind bekannt; diese erstreckt sich aber uber viele Stunden, ja bei Kaltblütern iiber mehrere Tage und in einzelnen Fällen über ein ganzes Jahr. Die vorliegenden Beobachtungen haben aber nur den Werth von Augenblicksbildern. Was dazwischen liegt, kann wohl erschlossen, aber auch falsch gedeutet werden.

Die directe Beobachtung ist tnerlässlich. Nun ist es klar, dass diese directe Beobachtung an den inneren Organen von Wirbelthieren vicht ausgeführt werden kann. Wenn auch bei erhaltener Circulation die natürlichen Vorgänge eine Zeit lang nahezu in gewohnter Weise ablaufen mögen, so wird doch die unvermeidliche Hervorzerrung der beobachteten Organe aus geschützten Körperhöhlen, die Entblössung von der deckenden Haut alsbald von Entzündung gefolgt werden. Solche Beobachtungen dürfen somit für die Construction des cyclischen Ablaufs normaler Veräderungen nicht ohne Weiteres verwerthet werden.

Man kennt bis jetzt die Veränderungen durch die Secretion nur an compacten Drüsen und weiss durch die Untersuchung erhärteter Organe, dass zu derselben Zeit in verschiedenen Regionen derselben Drüse verschiedene Zustïnde der Zellen sich finden können, so dass man den einen Zustand aus dem andern mit absoluter Gewissheit nicht ableiten kann.

Günstiger für die Entscheidung wären schon die vereinzelten Drïsen der durchsichtigen Nick- oder Schwimmhaut des Frosehes. Versuche, beim curarisirten Frosch die Nickhautdriisen von der Mundhöhle nach Enueleirung des Auges, oder die Drüsen der ausgebreiteten Schwimmbäute direct zu beobachten, bieten keine technischen Schwierigkeiten; aber es wollte mir nicht gelingen, an diesen vielzelligen Drüsen eine gentigende Sicherheit $z \mathbf{u}$ gewinnen. Ueberhaupt wird in Bezug auf die Frage, ob die Zelle befähigt sei, des Oefteren Secrete zu bilden und wieder abzugeben, die Ungewissheit so lange bestehen bleiben, als es sich um die Beobachtung zusammengesetzter oder auch einfacher aber vielzelliger 
Drüsen handelt. Hier sind die Veränderungen an einer und derselben Zelle unmöglich zu verfolgen.

Eine absolute Garantie wäre geboten, wenn an einer binreichend grossen und isolirten einzelligen Drüse die Veränderungen durch die Secretion bekannt wären: wenn durch künstliche Reizung beliebig oft das Secret aus der Drüse entfernt und in derselben Zelle von Neuem gebildet würde. So sind wir auf Thiere angewiesen, deren Leib durchsichtig genug ist und bei seiner Kleinheit eine längere fortgesetzte Beobachtung, unter dem Mikroskop gestattet, ohne dass der Zusammenhang der Theile in irgend welcher Art gestört wird.

Meine auf dem internationalen Aerzte-Congress zu London ausgesprochene Absicht, an marinen Copepoden den Secretionsvorgang einzelliger Drüsen zu studiren, hat sich nicht verwirklicht. Nach Bunn zurückgekehrt, war ich somit auf Süsswasserthiere angewiesen. Auf den Argulus foliaceus hatte ich schon lange, aber vergebens Jagd gemacht; bis ich zu Ende des vergangenen Herbstes durch die Gute des Herrn von la Valette St. George mit einer grossen Anzall dieser prächtigen Thiere beschenkt warde.

Die Anatomie des Argulus foliaceus ist von Ley $\mathrm{dig}^{\prime}$ ) and $\mathrm{CJaus}^{2}$ ) in erschöpfender Weise behandelt worden.

Die einzelligen Driisen sind im Mantel, den Beinen und in den Schwanzflossen gelegen und alle der directen Beobachtung zugänglich. Am günstigsten für die Zwecke einer längere Zeit fortzusetzenden Untersuchung sind die Drüsen in der Schwanzflosse jüngerer Weibchen. Bei den Männchen nehmen die Hoden einen grossen Theil der Flosse ein, und älere Weibchen sind zu dick, um eine Beobachtung mit stärkeren Linsen zu gestatten.

In der Schwanzflosse sind nicht alle Drüsen von derselben Grösse. Die Ausführungsgänge sind runde Röhrchen, oft von bedeatender Länge. Zellen und Ausführungsgänge sind von einer kernhaltigen Membran umzogen, so dass das Blut in einem vielbuchtigen Sinus circulirt, in den, wie in die Bauchhöhle der Wirbelthiere die Eingeweide, die einzelnen Drüsen hineinragen. An der Peripherie der Flosse bleibt eine breite Bahn für die Circulation der Blutflüssigkeit frei; hier ragt zuweilen eine Drusen-

1) Leydig: Zeitschr. für wissenschaftl. Zoologie. Bd. II.

2) Claus: Ibid. Bd. XXV. 
zelle hinein: ein Umstand, der für die Beobachtung einer bestimmten Zelle nicht ohne Vortheil ist. Man kann sich aber auch anderweitig hinlänglich sichern, bei wiederholter Untersuchung desselben Thieres stets dieselbe Drüsenzelle wieder aufzufinden, so dass die Wahl nicht schwer fällt.

Nimmt man einen Argulus frisch von seinem Fischwirth, so sind die grössten einzelligen Drüsen von einem zarten strahligen Gefüge. Vom langgestreckten Lumen des Ausführungsganges ziehen meridionale feine Strassen bis fast an den entgegengesetzten Pol der Driisenzelle, wo der mononucleoläre Kern sich findet. In den Interstitien der feinen helleren Strassen liegen dicht gereihte Körn$\mathrm{c}^{\text {hen: }}$ das Secretionsmaterial. In Wasser verquellen die isolirten Zellen; die Granula verschwinden. Auf Zusatz vou Ueberosmiumsäure zum frischen Präparat tritt intensive Brïunung der Granula ein, so dass man wohl annehmen darf, das Secret sei von specifischer giftiger Wirkung, wie bei den grossen Rückendrüsen der Salamandra maculosa, deren Granula sich ebenfalls intensiv in Ueberosmiumsäure schwärzen; Schleimzellen bleiben überall ungefärbt.

Reizt man einen Argulus mit Inductionsschlägen, die einen Froschmuskel eben zur Contraction bringen, so entleert sich das Secret. Es stürzt bei Beginn der Reizung, wie Pulverdampt aus einem Geschiitzrohr, aus dem Ausfiubrungsgange hervor: Eine continuirliche Secretion ist nicht zu erzielen. Dies sieht man am besten an den seitlich mündenden Drüsen des Mantels. Unterbricht man die Reizung, erschlafft die Körpermuskulatur, so tritt auf neue Reizung neuer Tetanus der quergestreiften Muskulatur und im Beginn desselben erneute Ausstossung von Secret aus den Drüsenzellen ein. Der Tetauus ist niemals so heftig, dass das Thier zwischendurch nicht spontane Bewegungen macht.

In einigen Fällen sieht man bei electrischer Reizung eine offenbare Contraction der Driisensubstanz eintreten; in vielen anderen Fällen bleibt dieselbe jedoch aus, und die Zelle wird nur durch die Contraction der quergestreiften Muskeln gleichsam ausgepresst. Die Schwanzflosse besitzt eine grosse Zabl von quergestreiften Muskelfasern, wie es Claus in Figur 41, Tafel 18 der Zeitschrift für wissenschaftl. Zoologie Bd. 25 abgebildet hat. Nebeu den in der Längsrichtung der Flosse verlaufenden Muskelfasern gibt es noch andere, die man am lebenden Thier nicht 
ohne Weiteres als solche erkennen kann. Diese an die kerntragende Membran der Drüsenzellen herantretenden Muskelfasern haben einen zur Längsachse der Schwanzflosse senkrechten oder auch schräg gebogenen Verlauf. Man sieht am unverletzten Thier wohl die Contractionen und die Kerne dieser eigenartigen, im optischen Querschnitt siegelringähnlich erscheinenden Fasern; ihre Querstreifung, nur an einer Seite der Muskelfaser vorhanden, kann aber erst an Zerzupfungspräparaten erkannt werden. In $0,6 \%$ Kochsalzlösung führen die isolirten Fasern noch lange Zeit spontane rhythmische Bewegungen aus.

Somit ist es nicht möglich, für den Act der Ausstossung des Secretes immer eine Contraction der Zellsubstanz verantwortlich zu. machen, obschon die Driisenzellen des Argulus zu gewissen Zeiten ganz sicher contractil sind. Man sieht auch, wie Claus dies schon hervorgehoben, zuweilen am ungereizten Thier buchtige Drïsen; zu anderen Zeiten nur glattrandige ${ }^{\mathbf{1}}$ ).

Hat man durch öfter wiederholte kurzdauernde Reizung die Drüse erschöpft, so dass kein Secret mehr zu erhalten ist, so bleibt der Kern der Zelle unverändert mononucleolär, wie es zu Anfang der Reizung war.

1) Ueber die Beobachtungen von Stricker und Spina an den Hautdrüsen des Frosches und ihre Tragweite für den Mechanismus der Secretion hat sich schon Heidenhain ausgesprochen. Es ist nicht möglich, den ganzen Ablauf der Secretion von einem Gesichtspunkt aus erklären zu wollen. Vor Allem ist bei den secernirenden Drüsen der Act der Bildung des Secretes und seiner Vorstufen scharf zu trennen von dem Moment der Ausstossung, den man nicht selten mit dem Namen "Secretion" belegt hat. Die morphologischen Veränderungen der Drüsen zur Zeit der Aufspeicherung und nach der Entleerung des Secretes beruhen auf der schaffenden Thätigkeit des Protoplasmas und dürfen nicht zusammengeworfen werden mit den StrickerSpina'schen Beobachtungen über Protoplasmabewegung in Drüsenzellen während der Ausstossung des Secrets.

Gewiss, werden innere Verschiebungen des Protoplasmas der Drüsenzellen bei der Entleerung des Secretes eine grosse Rolle spielen können. Nur so viel scheint gewiss zu sein, dass neben den von Stricker und Spina beobachteten Contractionen und amoeboiden Bewegungen noch viele andere Vorgänge concurriren, um die Secrete aus den Zellen fortzuschaffen. Die Muskeln der Drüsen, die verschiedenen Druckhöhen, unter denen die Secrete der einzelnen Drüsen aus den Ausführungsgängen abfliessen, weisen darauf hin. 
Die Strassenbildung und die Granula in der Zelle sind aber geschwunden und das gelblich opake Aussehen der Zellen hat einem durchsichtigen Platz gemacht. Bringt man jetzt das Thier an einen Fisch zurück, so ist nach höchstens drei Tagen die Zelle mit Secret wieder angefüllt and hat ihr ursprüngliches Gepräge wieder gewonnen. Neue Reizung wird das Secret nochmals entziehen, und die Ruhepause es in der Zelle wieder regeneriren. In einem günstigen Falle konnte ein und dieselbe Zelle vierzehn Tage lang beobachtet werden; das Thier wurde in dieser Zeit vier Mal 15 bis 25 Minuten lang discontinuirlich electrisch gereizt. Dieselbe Zelle hat $3 \mathrm{Mal}$ neues Secret gebildet. Einige Tage nach der vierten Reizung ging das Thier zu Grunde.

Länger als einen Monat liessen sich die Arguli überhaupt nicht am Leben erhalten; obwohl sie theils in fliessendem Wasser, theils in vortrefflichen Aquarien an kleinen Cyprinoiden gehalten wurden.

$\mathrm{Zu}$ diesen directen, am lebenden Object angestellten Beobachtungen dürften die folgenden an gehärtetem Material gewonnenen eine brauchbare Ergänznng liefern. An den Drüsen des Argulus foliaceus konnte wohl die wiederholte Neubildnng von Secretionsmaterial verfolgt werden; allein es wollte nicht gelingen, die Lebensdauer einer Zelle und die Art ihres Ersatzes zu controliren. $\mathrm{Zu}$ diesem Zwecke wurden erwachsene Salamander (Salamandra maculosa), die ich in grosser Zahl der Freundlichkeit des Herm Dr. Pfitzner verdankte, in der oben p. 298 geschilderten Weise gefüttert.

Die hier gewonnenen Resultate konnten durch ähnliche Erscheinungen bei anderen Thieren bestitigt werden.

Es zeigten sich num in den Magendrüsen von Salanandra und von Lacerta, im Pancreas von Salamandra Zellen, die in toto ausgestossen werden sollten.

Während die benachbarten Zellen von Fermentgranula erfüllt waren, waren diese durch den absoluten Mangel an Granula ausgezeichnet und durch einen Kern, der sich wesentlich von dem Verhalten normaler Kerne unterschied. Der lebeuskräftige Zellkern ist in frischem Zustande glänzend und wird in Ueberosmium- 
säure leicht gebräunt; die Kernkörperchen bewahren auch in diesem Reagens einen lebhaften Glanz. Die Kerne der abgestorbenen Drüsenzellen sind matt von Aussehen, sie bleiben in Ueberosmiumsäure hell; die in ihrem Inneren entbaltenen Körnchen werden mattschwarz. Die hier beschriebenen Zellen sind, so lange sie noch in dem Gefüge der ubrigen Drüsenzellen verblieben, stark vergrössert. Ihr Leib enthält eine in Ueberosmiumsäure sich absolut nicht färbende Substanz. Die Nachbarzellen sind stark comprimirt. Neben den noch an der Membrana propria und den Nachbarzellen haftenden abgestorbenen Zellen kommen weiter solche vor, die sich mehr oder weniger ans dem Zusammenhange gelockert haben; andere, die an tieferen oder höheren Punkten des Ausführungsganges frei im Lumen desselben liegen. Der Leib der Zelle wird mit dem Vorruicken im Ausfïhrungsgange kleiner und der ganze Zellenrest in Ueberosmiunsäure mehr und mehr tingirbar. Drïsen, deren Ausführungsgänge oder secernirende Schläuche solche Zellen enthalten, sind in ihren Endabschnitten -- dem Grunde der Schläuche - durch eine im Lumen enthaltene farblose Masse stark gebläbt.

Die Zeit des Auftretens dieses Absterbeprocesses der Zellen faillt in die Phase der lebhaftesten Verdaung und in die erste Periode der Regeneration des verbranchten Secretionsmaterials.

Während der Verdauung ist die Magenschleimhaut von Talpa europaea mit melurfachen Lagen einer gerinnbaren Substanz bedeckt. Zwischen den einzelnen Schichten dieser Masse sind ausgestossene Zellen eingeschlossen. An eine mechanische Entleerung aus den Drüsenschläuchen bei der Präparation ist nicht zu denken, und dic Beobachtung ausgestossener Zellen im Lumen der secernirenden Drüsen niederer Wirbelthiere (Salamandra, Lacerta) spricht dagegen, dass diese Zellen ausschliesslich vom Obertlächenepithel des Magens abstammen sollten.

Seitdem die Kernfiguren in den sich theilenden Zellen bekannt geworden sind, hat man auch in den Darmdrüsen erwachsener Thiere diese Bildungen nachgewiesen (Pfitzner, Gaule).

Es kam mir darauf an, die Zeit zu bestimmen, wann dieser Process der Zelltheilung sich vollziehe, und an welcher Art von Zellen er ablaufe.

Die Fütterungsversuche an Salamandra ergahen, dass längeres Hungern geeignet ist, eine enorme Vermehrung der im Darm vor- 
handenen Drïsenzellen in der ersten Zeit nach der Nahrungsaufnahme herbeizuführen. Das Keimlager der Oesophagusepithelien, die Magendrüsen und namentlich der von Eberth' ${ }^{1}$ ) beschriebene Ueberzug der Leber waren voll von Kerntheilungsfiguren. $\mathrm{Zu}$ anderen Zeiten sind die Kerntheilungsfiguren sehr selten; sie konnten in zahlreichen Schnitten von Oesophagus, Magen, Pancreas und Leber von $50 \mathrm{zu}$ verschiedenen Zeiten nach der Fitterung getödteten Salamandern nicht nachgewiesen werden. Durch den Ablauf einer einzigen normalen Secretionsperiode werden die Zellen also ihre Lebenskraft nicht einbüssen, wie dies auch durch die Beobachtung an den Drüsenzellen des Argulus erhärtet wird. Da aber Kern- und Zelltheilungen in Drïsen vorkommen, so fragt es sich, woher der Ersatz des Abgängigen geleistet wird. In den Drüsen sind die Zellen nur in einfacher Lage angeordnet; die Art des Ersatzes muss demnach eine andere sein als bei den geschichteten Epithelien, wo die unteren Lagen nicht über den embryonalen Charakter hinauskommen. Im Oesophagus finden sich die Kerntheilungsfiguren nur in dem Lager polygonaler Zellen unterhalb der Becher- und Flimmerzellen; in der Epidermis, der Cornea und den Nickhäuten der Amphibien vorzugsweise in der tiefsten und selten noch in der darauffolgenden Zellschicht.

In den Drüsen bewahrt jede einzelne Zelle die Fähigkeit der Reproduction, wenn sie auch schon functionirt hat. Es existirt somit zwischen den Drüsenepithelien und den in Lagen geschichteten Epithelien derselbe Unterschied, anf den ich in einer früheren Abhandlung hingewiesen, als es sich um den Vergleich der Fortpflanzung bei den Protozoen und den Metazoen handelte ${ }^{2}$ ).

In dem Pancreas von Salamandra maculosa sind zu keiner Zeit die Kerntheilungsfiguren so häufig als in den Magendrüsen dieses Thieres. Die Theilung vollzieht sich, wie das am Pancreas gerade recht dentlich nachgewiesen werden konnte, an Zellen, die mit den iibrigen in gleicher Flucht das Lumen begrenzen, and die in ihrer centralen Partie Secretionsmaterial abgelagert haben.

Wie die.Neubildung von Zellen eingeleitet werde, wird man sich wohl in folgender Weise erklären können. Die zur Elimi-

1) E b e r t h, d. Archiv, Bd. III.

2) Dies Arch. Bd. XVIII, pag. 97 und 98. - Das Keimlager der geschichteten Epithelien würde den Geschlechtsdrüsen der Metazoen entsprechen. 
nirung bestimmten Zellen sind gebläht; sie üben auf die Nachbarschaft einen in seiner Wirkung deutlich hervortretenden Druck aus. Vielleicht genügt dieser mechanische Reiz, die Nachbarzellen zur Theilung anzuregen, wie dies bei makroskopisch sichtbaren Neubildungen an geschichteten Epithelien hinlänglich bekannt ist. - Dass die Nachbarschaft auch bei geschichteten Epithelien Defecte zu decken im Stande ist, wäbrend im Allgemeinen und für gewöhnlich nur senkrecht aufstrebende Nachsehübe junger Zellen erfolgen, ist an der Benarbung grosser Wundfächen gut genug zu verfolgen. Was demgemäss für die geschichteten Epithelien das Keimlager, ist für die in einfacher Lage ausgebreiteten Drüsenzellen der protoplasmatische Fuss and der Kern der Zelle; von diesen geht jede Neubildung aus.

Da nun in den Drüsen nachgewiesenermaassen Zellen zu Grunde gehen und durch nene ersetzt werden, so wird man sich nach den Gründen zu fragen haben, wesshalb die Secretion etwas Anderes sei, als die Ausstossung der Geschlechtsstoffe oder die Abschuppung und Abstreifung der Oberflächenepithelien.

Wie die Verfolgung des Secretionsvorganges bei Argulus foliaceus zeigt, ist Secretion nicht identisch und fällt auch zeitlich nicht zusammen mit dem Absterben der Zellen.

Dass dem nicht so sei, geht ferner aus dem Bau der ausgestossenen Zellen des Pancreas und der Magendrüsen hervor: die Zellen haben sich stets, bevor sie ausgestossen werden, ihres specifischen Secrets entledigt.

Die Secretion besteht in der Bildung und Aufspeichernng der Vorstufen des Secretionsmaterials, seiner Umformung in den Zellen und in der Entleerung des fertigen Secretes aus den Zellen.

Wie alles Lebende aus uns unbekaunten Ursachen abstirbt und neuen Generationen Platz macht, so gehen auch nach einer gewissen Zeit Drüsenzellen zu Grunde und werden von lebenskräftigen Nachbarzellen ersetzt. Sterben alle Zellen gleichzeitig $a b$, so ist die Drüse vernichtet wie eine Protozoencolonie, von deren einzelnen einzelligen Organismen keiner mehr zur Theilung geschickt ist.

Secretion, Absterben und Vermehrung von Zellen sind zwei in gewissem Sinne völlig unabhängig von einander verlaufende Vorgänge.

Die Secretion mag wohl die Zelle abnutzen; die Zelle wird 
altern. Der Act der Secretion ist aber nicht gleichbedentend mit Zellentod; er ist eine energische Lebensbethätigung.

\section{Abschnitt.}

Von der Veränderung der Kerne ${ }^{1}$ ) und vom Nebenkern der Drüsenzellen.

Taf. XVII u. XVIII, Figg. 25, 27, 28;30-70.

Die neueren Arbeiten über den Zellkern haben eine Complicirtheit des Baues nacbgewiesen, die früher nicht geahnt worden war. Wenn an dieser Stelle der Gegenstand ebenfalls berührt wird, so geschieht es aus dem Grunde, eine Reibe einzeln schon bekannter Erscheinungen in zeitlicher Folge vorzuführen; frühere eigne Beobachtungen durch neue zu ergänzen, und durch weitere Mittheilung von Thatsachen Beiträge zu einer erweiterten Kenntniss vom Baue der Zelle und ihrer Bestandtheile zu liefern.

In den zahlreichen aus dem Breslauer physiologischen Institut hervorgegangenen Arbeiten über die histologischen Veränderungen in den Drisen wird auch auf eine constante ${ }^{2}$ ) Aenderung an den Kernen der Drüsenzellen hingewiesen. An Ảlcohol-Carminpräparaten sind die Zellenkerne der ruhenden Drüse zackig; sie werden rund und zeigen scharf hervortretende Kernkörperchen, sobald durch Nervenreizung die Drüse in 'Thätigkeit versetzt worden war. Aebnliche Erscheinungen hat $\mathrm{Hebold}^{3}$ ) an den Eileiterdriisen der geschwänzten Amphibien beobachtet.

Was die Erklärung dieses Verhaltens anlangt, so wird man

1) Vergleiche hierzu: Auerbach: Organologische Studien. Breslau 1874. - Strassburger: Zellbildung und Zelltheilung. 1. Auflage 1875. 3. Aufl. 1880. - Flemming: Beiträge zur Kenntniss der Zelle und ihrer Lebenserscbeinungen, d. Arch. Bd. XVI, XVIII, XX. - A uerbach, Strassburger und Flemming geben die Litteratur so vollständig, dass es wobl erlaubt ist, auf die ihren Arbeiten beigefügten Litteraturverzeichnisse zu verweisen. - R. Hertwig: Beiträge zu einer einheitlichon Auffassung der verschiedenen Kernformen. Morphol. Jahrb. Bd. II, p. 63.

2) Heidenhain: Hermann's Lehrbuch der Physiologie 1. c.

3) Hebold: Inaugural-Dissertation, Bonn 1879. 
nicht fehlgehen, wenn man annimmt, die Kerne in den rubenden Drüsenzellen seien durch das im Leib der Zellen angehäufte Secretionsmaterial mechanisch comprimirt und fänden erst nach der Entleerung des Secrets die Möglichkeit, sich wieder in eine ihnen zukommende Gleichgewichtslage zurückzuversetzen. Man findet nämlich nicht allein in den secernirenden Zellen, sondern in allen Zellen, die zeitweise sich mit Stoffen beladen und sich derselben entledigen, immer die von Heide $\mathrm{n}$ ba in hervorgehobene Erscheinung wiederkehren. In den früheren Abschnitten ist auf diese Eigenthümlichkeit der Kerne der Hauptzellen und der Schleimdrisenzellen aufmerksam gemacht worden; hier sei noch auf die Compression der Kerne in Fettzellen und ähnlichen mit Reservestoffen geftullten Zellen hingewiesen.

In den Hautdrisen des Argulus foliaceus sind die Zellenkerne in verschiedenen Zuständen; die einen enthalten während des Lebens nur ein Kernkörperchen, die anderen deren zwei und mehrere.

Reizt man eine Drüse mit mononucleolärem rundem oder ovalen saftigen Kern, so bleibt der Kern auch nach der Ausstossung des Secretes mononucleolär. Die Secretion kann demgemäss die Kerne verändern: die Kernveränderung ist aber keine wesentliche Erscheinung, da sie ebensogut fehlen kann.

Während diese Veränderungen also in einfachster Weise auf mechanischem Wege ihre Erklärung finden dïrten, möchte es vor der Hand nicht gerathen sein, andere gleich zu besprechende erklïren zu wollen. Wir mitssen uns begntigen, die Formen in ihrer natülichen Reibenfolge zu beschreiben und verzichten vorläufig auf jede Erklärung.

Auerbach hat in seinen organologischen Studien die eigenartige Erscheinung des sog. mono- und multinucleolären Zustandes der Zellenkerne genetisch verfolgt und dabei die wichtige Beobachtung gemacht, dass bei den Musciden zu Anfang und am Ende des Larvenlebens die Zellen der Speicheldrüsen und des Fettkörpers nur ein Kernkörperchen enthalten, während in der Zwischenzeit die Zahl der Kernkörperchen gewaltig vermehrt gewesen war. Es steht also die Beobachtung an Eiern nicht unvermittelt da, wo ebenfalls eine Vermehrung und Wiederabnahme von Kernkörperchen beobachtet worden ist.

Bei meinen Untersuchungen an den Darmdrüsen der Wirbelthiere und den Hautdrüsen des Argulus foliaceus war es auffallend, 
wie mono- und multinucleoläre Kerne nicht allein gleichzeitig neben einander vorkamen, sondern wie auch unter bestimmten Bedingungen die eine Art von Kernen vorwiegend war.

Es liess sich im Allgemeinen feststellen, dass während des ungestörten Ablaufs der Secretion die mononucleolären Kerne vorherrschten, dass nach längerem Hunger die multinucleolärev Kerne an Zabl vermehrt waren.

Beim Argulus foliaceus konnte sogar der Uebergang der einen Form in die andere beobachtet werden. Dazn erwiesen sich am geeignetesten die zweizelligen Drüsen in dem Blutraum der Saugscheiben. Die Drüsenzellen der Saugscheiben sind von Leỵdig und Claus (l. c.) schon beschrieben worden. Es gibt zwei Arten. Die eine liegt in dem Bauch des kelchfờrmigen Saugfusses; die andere, um die es sich hier handelt, unterhalb des Velum, welches durch Muskeln retrahirt und durch den Anprall der im Inneren circulirenden Blutfliussigkeit vorgestuilpt werden kann. Die Drüsenzellen sind stets zu zwei vereinigt ${ }^{1}$ ). Ihr Ausfibrungsgang ist schwer zu sehen; doch glaube ich ihn einmal beobachtet zu haben. Wo derselbe im Centrum der Berührungsfläche der beiden verbundenen Zellen beginnt, liegt eine von hellem Hof umgebene Figur radienartig in beide Zellen hineinragender Strahlen.

Bei den zahlreichen zur Untersuchung verwandten Arguli fanden sich regelmässig in den Drüsen kräftiger Thiere, und wenn sie eben aus den Teichen eingefangen waren, vorwiegend mononucleoläre Kerne. Verfolgte man eine bestimmte Zelle eine längere Zeit hindurch, so verwandelte sich allmählich der mononucleolïre Zustand in den polynucleolären und wenn dieser eine Zeit lang. bestanden hatte, ging manche der polynucleolär gewordenen Zellen zu Grunde. Diese Beobachtungen sind vielfach wiederholt angestellt worden.

Ein Drüsenzellenpaar der Saugscheibe von Argulus foliaceus hatte am 12. October mononucleoläre Kerne; am 18. October zeigten sich viele Kernkörperchen in Kern; nach und nach ging die Granulirung der Zellen, die Strahlung verloren und die Kerne waren

1) Zweizellige Drüsen hat jüngst Andreae von Sipunculus nudus beschrieben (Zeitschr. für wissenschaftl. Zoologie Bd. 36, p. 214); zwei- and mehrkernige Drüsenzellen Max Weber vom Hepatopancreas der Crustaceen (d. Arch. Bd. 17, p. 402). 
platte Ovoide mit mehreren glanzlosen Körperchen darin. In der einen Zelle umgab schliesslich nicht einmal eine gemeinschaftliche Hülle die Reste des Kernes. Nachdem dieser Zustand seit dem 25. October angedauert, ging das Thier am 6. November zu Grunde. Unterdessen waren auch die anderen Drtisenzellenkerne sowohl in dem beobachteten Quadranten als in den ubrigen beiden Saugscheiben entweder polynucleolär geworden, oder die Zellen hatten die oben beschriebene regressive Metamorphose mehr oder weniger durchgemacht.

Der multinucleoläre Zustand des Kernes bezeichnet demgemäss einen Wendepunkt im Zellleben. $\mathrm{Da}$ bei den Eiern und nach Auerbach's Beobachtungen auch an anderen Zellen der polynucleoläre $\mathrm{Zustand}$ in den mononucleolären zurückgeführt werden kann; da nach meinen Beobachtungen der polynucleoläre $\mathrm{Zu}$ stand des Kernes dem Tode der Zelle voraufgeht; da weiter der polynucleoläre Zustand des Kernes aus dem mononucleolären sich entwickelt, so wird man den Kern mit vielen Kernkörperchen als den Ausdruck einer Ruhepause der Kernfunctionen auffassen können, die entweder zum krïftigen Leben oder zum Tode iberleitet. Die Zellen, welche aus den Drüsen ausgestossen werden sollen, die Zellen in den abgestossenen Epidermisschichten haben polynucleoläre Kerne; in den Ganglienzellen findet sich nur ein Kernkörperchen.

Diese Erscheinungen spielen sich an jeden Zellkern ab. Soll der Kern und mit ihm die Zelle sich theilen, so kann dies, soweit wir bis jetzt wissen, in zweifacher Weise geschehen. Der eine dieser Vorgänge ist erst in den letzten zehn Jahren bekannt geworden und besonders von Strassburger und Flemming sorgfältig studirt; der andere wird von vielen Histologen ganz geleugnet, obwohl er der früher ausschliesslich bekannte war.

Es handelt sich um die sogenannte indirecte und directe Kerntheilung, eine Theilung mit oder obne voraufgehende Bildung eines Fadenapparates im Kern.

Im XX. Band dieses Archivs hat Henle der directen Kerntheilung nicht ganz mit Recht den Boden zu entziehen versucht. Die bisher beschriebenen Formen entstehen nicht allein unter der Einwirkung von Säuren, sondern sind auch in den überlebenden Geweben schon vorhanden. Wenn man, wie dies von la Valette 
St. George $\left.{ }^{1}\right)$ gezeigt, die Entwicklungsstadien der Spermatosomen verfolgt, so findet man die maulbeerförmigen Kerne bei der Untersuchung des frischen im Humor aqueus zerzupften Hodens. Meine eigenen Angaben ${ }^{2}$ ) über das Vorkommen der maulbeerförmigen Kerntheilung in den Geschlechtsdrüsen der Embryonen und erwachsenen Thiere sind ebenfalls auf Untersuchung der frischen Organe zum grössten Theil basirt. An der in Humor aqueus des Thieres ïberlebenden Cornea von Salamandra und Rana kann man die eingeschnürten Kerne nicht allein im tiefsten Zellenstratum beobachten, sondern auch bei Reizungsvorgängen an den Wanderzellen zwischen den Epithelien alle Uebergangsstadien von dem vielfach eingeschnürten bis zum vielfach getheilten Kern. Daneben kommen auch Kernfiguren vor. In meiner Arbeit über die Differenzirung des Geschlechts ${ }^{3}$ ) sind beide Vorgänge beschrieben und vielfach abgebildet. Aehnliches berichtet $\mathrm{Klein}{ }^{4}$ ) von der Epidermis der Amphibien.

Bezliglich der maulbeerförmigen Kerntheilung in den Spermatogonien kann ich der von $\mathrm{Krause}{ }^{5}$ ) geäusserten Meinung. nicht beipflichten. Die maulbeerförmige Kerntheilung tritt nicht gegen das Ende der Samenkörperentwicklung auf, sondern am Anfang. Ein Cystenkern in regressiver Metamorphose ist auf Taf. III Bd. $18 \mathrm{~d}$. Arch. abgebildet; an diesem ist von maulbeerförmiger Theilung Nichts zu sehen, wohl aber an der unter der abgängigen Cyste in ihrer Follikelhaut gelegenen Spermatogonie, aus der sich demnächst neue Samenkörper entwickelt haben würden. - Es würde zn weit führen, die Angaben $\mathrm{K}$ rause's in extenso zu besprechen, die im Allgemeinen eine Bestätigung des Entwicklungsgesetzes der Samenkörper enthalten, wie es von la Valette St. George aufgestellt hat; nur möchte ich auf den einen Punkt hinweisen, dass "Spermatogonie" (von la Valette $S$ t. George) nicht mit "Fusskern der Spermatoblasten" (Krause) identificirt werden darf.

1) D. Arch. Bd. XII, Taf. 34 Fig. 8 und a. a. 0.

2) D. Arch. Bd. XVIII.

3) D. Arch. Bd. XVIII.

4) Quarterly Journal of microscop. science, 1879.

5) W. Krause: Handbuch der menschlichen Anatomie; Nachträge zum 1. Bd. 1881.

Archiv f. mikrosk, Anatomie, Bd, 21. 
Vielleicht ist in Folge dieser von $\mathrm{Krause}$ adoptirten abweichenden Auffassung die Deutung der manlbeerförmigen Kerntheilung als eines Absterbungsphänomens zu erklären.

Ein mustergültiges Object für das Studium der Beschaffenheit des Zellkernes ist der von Eberth ${ }^{1}$ ) zuerst beschriebene und von ihm als lymphoides Gewebe gedeutete Ueberzug der Salamanderleber. Es finden sich dort mono- und polynucleoläre Kerne, Kerne in allen Stadien der directen und indirecten Kerntheilung. Die indirecte Kerntheilung ist nach einer ausgiebigen Fütterung die vorwiegende. Die eingeschnürten Kerne, Zellen mit drei bis fünf deutlich abgegrenzten Kernen sind zu allen Zeiten reichlich vorhanden.

Wenn sich die Natur des Leberüberzuges bei Salamandra maculosa als eines lymphkörperbildenden Organes bestätigen sollte, so wäre auch für die farblosen Blutkörperchen eine indirecte Kerntheilung nachgewiesen. Daneben besteht aber auch eine directe Kerntheilung, von der es allerdings vorlänfig noch nicht mit derselben Sicherheit durch die Benbachtung festgestellt ist, ob sie von einer Zelltheilung gefolgt werde. Doch machen die Beobachtungen an den Geschlechtsdruisen dies sehr wahrscheinlich. Dass aber eine Zelle nicht absterbe, wenn oder weil sie mehrere Kerne enthalte, dürfte aus den Untersuchungen $W$ ebers${ }^{2}$ ) an dem Hepatopanereas der Crustaceen hervorgehen. Auch in den Epithelien des Darmes habe ich bei Armadillo öfters zwei Kerne in derselben Zelle gesehen.

Ob nun, wie Elias Metschnikoff ${ }^{3}$ ) anzunehmen scheint, die sogenannte directe und indirecte Kerntheilung nur Phasen eines zusammengehörigen complicirten Processes seien, kann vorläufig nicht mit Sicherheit entschieden werden; nur soviel ist gewiss, dass die auch Fragmentation genannte, maulbeerförmige oder directe Kerntheilung zu einer ächten Theilung filhrt und keine regressive Metamorphose darstellt.

Das Gesagte zusammenfassend, wilde man bei den verschiedenen Kernformen von dem mononucleolären Zustande auszugehen haben; dieser wird in den polynucleolären übergeführt. Stirbt die Zelle $a b$, so zcrfällt der Kern zuweilen ganz. Zu einer Vermeh-

1) D. Arch. Bd. III.

2) D. Arch. Bd. XVII, p. 385 sqq, besonders p. 402.

3) Zeitschr. für wissenschaftl. Zoologie, Bd. 36, p. 433. 
rung von Zellen bedarf es einer vorgängigen Theilung des Kernes, die, soweit dies durch continuirliche Beobachtung bis jetıt hat festgestellt werden können, durch die Bildung eines eigenthümlichen Fadenapparates im Kern eingeleitet wird. Neben dieser indirecten Kerntheilung kommt auch eine directe in Folge einer Durchschnurung des Kernes vor. Dass diese Form der Kerntheilung zu einer ächten Theilung von Zellen führe, ist wahrscheinlich.

Wenn oben von mono- und polynucleolären Kernen die Rede war, so ist diese Auerbach entlehnte Bezeichnung auch aus dem Grunde beibehalten worden, weil an dem lebenden Object keine weitere Struktur an den Zellkernen wahrzunehmen war. Es kommen auch vernetzte Kerne vor; die sog. Substanz des Kernes kann sich vermöge ihrer amöboiden Bewegungsfähigkeit in die verschiedensten Gestalten umgiessen. Doch wird man nicht berechtigt sein, die durch Reagentien neben den Kernkörperchen hervorgerufenen Netze in den Kernen als präexistirend anzusehen; weil die wirklichen tingirbaren Netze, von der zartesten Form bis zur breitfadigen Kernfigur, auch im frischen Zustande gesehen werden können.

Schliesslich möchte ich eines Gebildes Erwähnung thın, dessen Entstehungsgeschichte und Bedeutung für das Leben der Zelle ich bis jetzt vergeblich aufzuklären versucht habe. Fortgesetzte Untersuchungen werden vielleicht zum Ziele ftihren.

Es finden sich nämlich im Pancreas von Salamandra maculosa und zwar in dem nicht von Secretionsmaterial erfïllten Theile der Zellen, zwischen Kern und Membrana propria, eigenartige Gebilde, die nicht zu allen Zeiten gleich bäufig und gleich gestaltet sind, denen ich vorläufig ohne Präjudiz den Namen „Nebenkern“ beilegen möchte.

Der Nebenkern ist entweder solitär oder multipel, solid oval oder spiralig gedreht, oft auch lockig gewunden. Der solitäre Nebenkern ist grösser als viele gleichzeitig in einer Zelle, vorhandenen einzeln genommen. Im frischen unter Zusatz von Jodserum oder Humor aqueus bereiteten Zerzupfungspräparat oder nach Maceration in verdünnter Chromsäure kann el isolirt werden und nimmt Farbstoffe in sich auf. Man findet ihn in Zellen, deren Kerne mono- oder polynucleolär oder auch ganz regressiv sind. Am 4. bis 5. Tage nach einer Fütterung ist er fast in jeder Zelle des Pancreas vorhanden. In der ersten Zeit nach der Fuitterung 
wird man ihn schwer oder vielleicht gar nicht finden. In der Drîse längere Zeit hungernder Thiere ist er selten.

Ausser dem Pancreas des Salamanders zeigten auch 72 Stunden nach einer Fitterung die Oesophagealdrüsen von Rana, die des Secrets beraubten Drisen von Argulus, das Pancreas von Triton ächte Nebenkerne; die fadenartige Beschaffenheit konnte bei Triton ebenfalls constatirt werden. Die Nebenkerne der tibrigen angeführten Zellen waren anscheinend solid.

Vergleicht man den Nebenkern der Drüsenzellen mit anderweitig bekannten Bildungen, so wird man wegen des zeitweisen Auftretens an eine Art von Nervenendigung, wie sie Pfitzuer ${ }^{1}$ ) jüngst im Epithel von Froschlarven beschrieben hat, nicht denken können. Die Möglichkeit der Isolirung des Nebenkernes, sein der Zeit und der Zahl nach variables Vorkommen sprechen dagegen.

Man wird auch nicht leicht geneigt sein, den Nebenkern der Drüsenzellen mit solchen Einlagerungen in Zellen zu vergleichen, die zu einer totalen chemischen Umwandlung der Zelle führen, wie sie jüngst noch von Waldeyer ${ }^{2}$ ) an verhornenden Epithelien beschrieben worden sind.

Dagegen wird man den Nebenkern der Drusenzellen wohl mit dem von Wittich ${ }^{3}$ ) entdeckten Dotterkern der Eier, dem durch von la Valette St. George $\mathrm{e}^{4}$ ) zuerst bekannt gewordenen Nebenkern der Spermatocyten, den von Leydig ${ }^{5}$ ) aus der Epidermis von Pelobateslarven beschriebenen Bildungen ${ }^{6}$ ) in eine Categorie bringen dürfen.

Sicheren Aufschluss kann man jedoch erst dann erwarten, wenn die Entstehungsgeschichte dieser Gebilde in derselben Weise gefördert sein wird, wie es kürzlich für den Dotterkern ${ }^{7}$ ) geschehen ist.

1) Morphol. Jahrb. Bd. VII. p. 726.

2) Festgabe an Jacob Henle, Bonn 1882, p. 141-163.

3) von Wittich: Inaugural-Dissertation, Halle 1845.

4) D. Archiv, Bd. III, 1867 .

5) Neúe Beiträge zur anatomischen Kenntniss der Hautdecke und Hautsinnesorgane der Fische. Halle 1879.

6) Die von Leydig gegebenen Zeichnungen erlauben nicht, den Einlagerungen in den Zellen dieselbe Bedeutung zu geben wie sie Pfitzner für die von Eberth gemachten Angaben (d. Arch. Bd. II) versucht hat.

7) Schütz: Ueber den Dotterkern etc. Inaug.-Dissert. Bonn 1882. 


\section{Erklärung der Abbildungen auf Tafel XV-XVIII.}

(Sämmtliche Figuren sind mit der Camera lucida in der Höhe des Mikroskoptisches gezeichnet.)

Tafel XV.

Fig. 1. Der untere Theil einer in Jodserum isolirten und dann in $1 \%$ Ueberosmiumsäure gehärteten, in Glycerin aufbewahrten Labdrüse der grossen Curvatur des Magens von Lepus cuniculus. Die Färbùngsunterschiede in Ueberosmiumsäure sind nicht so gross, wie in Fig. 2. h Hauptzelle, b Belegzelle. Zeiss CC, Oc. II.

Fig. 2. Unterer Theil einer Labdrüse aus dem Fundus desselben Kaninchenmagens, in derselben Weise behandelt. In den Hauptzellen lagen vor der Einwirkung der Ueberosmiumsäure grosse Granula, nach deren Auflösung die verzerrten Kerne in den Zellen erst sichtbar werden. h Hauptzelle, b Belegzelle. Zeiss CC, Oc. II.

Fig. 3. Eine Drüse mit Belegzelle (b) aus dem Pylorustheil der Magrenschleimhaut von Vesperugo serotinus. Zeiss CC, Oc. III.

Fig. 4. Unteres Ende einer Magendrüse aus dem mittleren Theile der Magenschleimhaut einer $2^{2} / 2$ Stunden zuvor gefïtterten Talpa europaea. Osmiumsäurepräparat. Zeiss F, Oc. I. h Hauptzelle, b Belegzelle.

Fig. 5. Labdrüse von Vesperugo serotinus aus dem Cardialtheile (Fundus) der Magenschleimhaut. Osmiumsäurepräparat. Zeiss CC, Oc. III. h Hauptzelle, b Belegzelle.

Fig. 6. Untere Parthie einer Labdrüse von Talpa europaea (2 Tage Hunger.) Osmiumsäurepräparat. Zeiss F, Oc. I. Die Belegzellen sind kleiner als in Figur 4; manche sind stark gebräunt und enthalten keine Granula. h Hauptzelle, b Belegzelle.

Fig. 7. Blindes Ende eines Pankreasdrüsenschlauches von Cyprinus carpio. - Magen leer. - In den Zellen centrale Körnerzone. Osmiumsäurepräparat. Zeiss F, Oc. I.

Fig. 8. Zum Bau des Pancreas von Salamandra maculosa. Schnittpräparat parallel zur Oberfläche einer in Osmiumsäure erhärteten Drüse. In den Ausführungsgang münden zahlreiche verästigte Schläuche. Zeiss CC, Oc. II. A Ausführungsgang mit den warzenartigen Zellen.

Fig. 9. Bau der pancreatischen Drüsenzellen von Salamandra maculosa (48 Stunden nach der Fütterung). Auf die centrale Körnerzone folgt 
der Kern und zwischen diesem und der Membrana propria des Drüsenschlauches der Nebenkern. Die Zelle war frisch in Jodserum isolirt.

Fig. 10. Zwei Drüsen aus dem zweiten Abschnitt der Magenschleimhaut von Anguis fragilis. Zeiss F, Oc. I.

Fig. 11. Unteres Ende einer Labdrïse aus dem Fundus des hungernden Kaninchens nach Einwirkung von Ueberosmiumsätre. Zwischen den der Granula beraubten Hauptzellen (h) mit verzogenen Kernen drei Belegzellen (b) mit runden oder ovalen mononucleolären Kernen und fein granulirtem Zellleib. Zeiss F, Oc. II. (Das nach Auflösung der Fermentgranula sichtbare Filigran in den Hauptzellen ist in der Lithographie zu hart ausgefallen.)

Fig. 12. Labdrüse aus dem Magenfundus von Vesperugo Nathusii. Osmiumsäurepräparat. Die Granula der Hauptzellen (h) sind erhalten und können ungefähr als Paradigma dieser Granula in den frischen Hauptzellen anderer Säugethiere dienen. Die meisten Zellen sind total mit den groben Körnern gefüllt, andere nur in der centralen Zone. Die Belegzellen (b) sind feinkörniger; in manchen Fällen ist eine zum Lumen des Schlauches radiär gestellte Anordnung der feinen Granula in den Belegzellen zu erkennen. Die Kerne der Hauptzellen sind mononucleolär (vgl. Figur 5); manche Kerne der Belegzellen polynucleolär. Zeiss F, Oc. II. (Der Magen des Thieres enthielt einige Haare.)

\section{Taf. XVI.}

Fig. 13. Drüse mit Ausführungsgang (A) aus dem in Ueberosmiumsäuro erhärteten Oesophagus von Rana esculenta. Zeiss BB, Oc. I. (M Mündung des Ausführungsgangs.)

Fig. 14. Eine Drüse aus dem ersten Magenabschnitt von Lacerta agilis; vier Stunden nach der Fütterung. Osmiumsäurepräparat. Zeiss $F$, Oc. II.

Fig. 15. Eine Drüse ebendaher von einem längere Zeit hungernden Thiere. Osmiumsäurepräparat. Zeiss F, Oc. II.

Fig. 16. Eine Drüse aus dem ersten Magenabschnitt von Anguis fragilis. Osmiumsäurepräparat. Zeiss F, Oc. II.

Fig. 17. Schleimdrüsen im Oesophagus von Anguis fragilis. Querschnitt durch die Wand des Oesophagus. Unter dem Epithel und nach Aussen von der Muscularis Pigmentzellen im Querschnitt. Osmiumsäurepräparat. Zeiss CC, Oc. I. Die Muscularis mucosae bildet Leisten in der Schleimhaut.

Fig. 18. Querschnitt einer Drüse aus dem Magen einer hungernden Emys europaea. Osmiumsätirepräparat. Zeiss F, Oc. I; rechts im Bild 
drei fein granulirte Zellen, Belegzellen; links fünf helle und eine ganz geschwärzte, in der keine Structur zu erkennen ist.

Fig. 19. Querschnitt einer Drüse aus dem zweiten Abschnitt des Magens von Lacerta agilis; radiäre Anordnung der Körnchen in den Belegzellen.

Fig. 20. Querschnitt durch Drüsen der grossen Curvatur von Cavia cobaya. - Osmiumsäure, Alcohol, Safranin. - h Hauptzelle, b Belegzelle. Zeiss F, Oc. 1 .

Fig. 21. Unteres Ende einer Fundusdrüse des Magens von Lepus cuniculus. - Osmiumsäure, Alcohol, Safranin. - Zeiss F, Oc. II. Das Filigran der Hauptzellen ist ziemlich naturgetren im Vergleich zu Figur 11 auf Taf. I; h Hauptzelle, b Belegzelle.

Fig. 22. Oberes Ende einer zusammengesetzten Labdrüse von Sus scrofa. Zeiss CC, Oc. I. (Die Belegzellen sind im Drüsenhalse nicht continuirlich aneinandergereiht; sie stossen an das Lumen an, und ihre Kerne sind meist polynucleolär); b Belegzelle.

Fig. 23. Querschnitt einer Magendrüse von Talpa europaea. Alcohol, Carminpräparat. h Haupt-, b Belegzelle.

Fig. 24. Unteres Ende einer Magendrüse vom hungernden Hund (1 Tag nach der Fütterung.) Zeiss CC, Oc. III. (Die Belegzellen b reichen auch hier an das Schlauchlumen heran; ihre Kerne sind meist mononucleolär.)

\section{Tafel XVII}

Fig. 25. Ungereizte mit Secretionsmaterial gefüllte Drüse aus der Schwanzflosse eines $\&$ Argulus foliaceus. Bei Zeiss F, Oc. II nach dem Leben gezeichnet. Der Ausführungsgang ist nur zum kleinen Theil dargestellt.

Fig. 26. Die zweite, tubulöse Form der Magendrüsen von Lacerta agilis. Zeiss BB, Oc. I. Osmiumpräparat. In der Lithographie treten die Färbungsunterschiede der beiden Zellenarten im Grunde der Schläuche nicht deutlich genug hervor.

Fig. 27. und 28. Dieselbe Drüse aus der Schwanzflosse eines $q$ Argulus foliaceus in verschiedenen Stadien. Bei Zeiss F, Oc. II nach dem Leben gezeichnet. Ausführungsgang nur zum Theil dargestellt; die bindegewebige Hülle nicht gezeichnet; in Fig. $27 \mathrm{n}$ Nebenkern; in Figur $28 \mathrm{~m}$ ein optischer Querschnitt der contractilen Elemente (quergestreifte Muskelfasern) zwischen den Drüsenzellen. Zur weiteren Erläuterung diene Folgendes: Die dargestellte Drüse hatte (25. November 1881) dsselbe Aussehen wie Fig. 25. Nach 10 Minuten langer intermittirender Reizung mit Inductionsschlägon nahm die Drüse das Aussehen Fig. 28 an; $1 \frac{1}{2}$ Stunden später war die starke Lappung der Oberfäche wieder verschwunden, die 30 Minuten nach 
dem Aufhören der Reizung noch vorhanden gewesen. Am folgenden Tag war die Granulirung der Zelle wieder kräftiger geworden; die Strassenbildung aber noch nicht in ibr zu sehen. Am 27. November wurde die Drüsenzelle wieder schön strassig; ihr Kern gross, blass; die Zahl der Kernkörperchen nicht zu bestimmen. (Der Kern war zu Anfang der Beobachtung mononucleolär gewesen.) Nach einer 10 Minuten langen electrischen Reizung trat die Buckelbildung an der Drüse wiederum auf; die Granulirung nahm ab und die Strassenbildung verschwand ganz. Am folgenden Tage (28. November) batte die Drüse ihr ursprüngliches Aussehen noch nicht wiedererlangt. Fig. 27 stellt die Drüse dar, wie sie sich in der abgeschnittenen Schwanzflosse am 28. November verhielt. Das Präparat wurde in Wasser untersucht; die Muskeln contrahirten sich nicht spontan, wie sie es vorher in andern auf gleiche Weise behandelten Präparaten gethan batten; die Schwanzflossen waren dicht oberhalb ihrer Insertion abgeschnitten.

Fig. 29. Magendrüsen der hungernden Forelle (Salmo fario) Dezember. Osmiumsäure. Zeiss CC, Oc. III.

Fig. 30. Ungereizte Hautdrüse (kleine Schleimdrüse) von Salamandra macu-

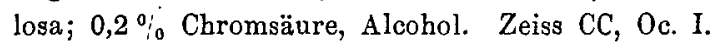

Fig. 31. Eine Hautdrüse (kleine Schleimdrüse) von derselben Salamandra maculosa nach kurzdauernder Secretion. Dieselbe Präparation und Vergrösserung wie in Fig. 30; im Inneren der Drüse ist eine geronnene Schleimwolke; umgeben ist die Drüse von einer feinen Muskellage und dichtem Pigment. Diese Aeusserlichkeiten der Umgebung sind in Fig. 30 nicht dargestellt.

Fig. 32. Schlauchquerschnitt einer Oesophagealdrüse (Rana esculenta, 71 Stunden nach der Fütterung getödtet.) Osmiumsäure, Hämatoxylin, Zeiss F, Oc. I. - Die Fermentgranula des frischen Präparats sind in der Ueberosmiumsäure fast total aus den Zellen geschwunden. In der Lithographie treten die Färbungsunterschiede nicht scharf genug hervor: die homogen erscheinenden Zellen mit mononucleolärem Kern sind tiefbraun durch die Ueberosmiumsäure gefärbt worden; die feinpunctirten mit mono- oder polynucleolären Kernen blieben hell.

Fig. 33. Aus einer Magendrüse (Pepsindrüse) von Rana esculenta. 71 Stunden nach der Fütterung. Zellen gross, Kerne mononucleolär; feine Granula in den Zellen. - Osmiumsäure. Zeiss F, Oc. I.

Fig. 34. Aus einer Magendrüse (Pepsindrüse) von Rana esculenta; 3 Wochen Hunger. (Winter 1880; das vorige Präparat, Fig. 33, Sommer 1881.) Zellen klein; die Kerne polynucleolär; Granula in den Zellen fehlen. - Osmiumsäure, Zeiss F, Oc. I. 
Fig. 35. Drüse aus der Schwanzflosse eines $\&$ Argulus foliaceus, 9 Stunden post mortem. Das Thier war vorher mehrere Male electrisch gereizt worden; die Drüse hatte in gewohnter Weise nach jeder Reizung Secret abgegeben und im Laufe von ein bis zwei Tagen wieder neugebildet. - Osmiumsäure. n Nebenkern dunkler gefärbt als der Kerrn. Zeiss F, Oc. II.

Fig. 36-39. Querschnitte durch je einen Schlauch der Eileiterdrüsen von Rana fusca zur Demonstration der Umwandlung der Kerne. Fig. 36. Habitus der Zellen und Kerne vom Hochsommer bis zur Laichzeit; Fig. 37 nach dem Laichen. Beide Figg. bei Zeiss CC, Oc. III gezeicbnet und mit Rücksicht. auf den schleimigen Inhalt der Zellen vor der Laichzeit beide Präparate in absolutem Alcohol gehärtet. Fig. 38 und 39 stellen Schlänche nach dem Laichen dar mit polyund mononucleolären Kernen. In Fig. 38 ist ausserdem die bekannte Fettkörnchenbildung in Zellen vorhanden. Die Figg. 38 und 39 sind bei Zeiss F, Oc. I gezeichnet.

Fig, 40 und 41. Aufeinanderfolgende Stadien von regressiver Metamorphose an einem Paar zweizelliger Drüsen aus der Saugscheibe von Argulus foliaceus (Fig. 40 vom 25. October, Fig. 41 vom 1. Novbr. 1881.) cf. Fig. 51 auf Taf. XVIII.

Tafel XVIII.

Fig. 42. Pancreas von Salamandra maculosa, Osmiumsäure, Serie III, Nr. 7. (120 Stunden nach der Fütterung.) Zeiss F, Oc. II. - Die Zelle x hat sich losgelöst und wird eliminirt; in y Vorbereitung zu diesem Process. $n$ Nebenkern in Hämatoxylin intensiv gefärbt.

Fig. 43. Ebendaher; Ansicht von oben auf das blinde Ende eines Drüsenschlauches. Osmiumsäure. Zeiss F, Oc. II. In den Zellen mononucleoläre, regressive und buchtige Kerne (o); letztere Art ist in Fig. 60 aus einem frischen Isolationspräparat abgebildet.

Fig. 44. Pancreas von Salamandra maculosa. 0,2\% Chromsäure, Alcohol, Safranin. (Serie I, 48 Stunden nach der Fütterung.) Aus einem Längsschnitt eines Drüsenschlauches. Bei a die Membrana propria; bei b das Lumen des Schlauches; in der mittleren Zelle eine Kernfigur; im Kopf aller Zellen Secretionsmaterial.

Fig. 45. Zellen der oberen Epidermisschicht von Triton taeniatus; regressive Metamorphose der Kerne. - 0,2 \% Chromsäure, Alcohol, Safranin.

Fig. 46. Aus der tiefsten Zellenlage einer infiltrirten Cornea von Rana esculenta; 0,2 \% Chromsäure, Alcchol, Safranin. Das Präparat zeigt 
einen mononucleolären, einen fein vernetzten und drei buchtige Kerne.

Fig. 47. Aus der näcbstfolgenden Zellenlage derselben Cornea.

Fig. 48. Zellen zwischen den Epithelien und in der Substanz derselben Cornea. (Präparation wie bei 46 und 47.) a mit zackigen Fortsätzen, also wohl amoeboid; b mit drei isolirten Kernen; c mit mebreren Kernen.

Fig. 49. und 50. Isolirte Kerne aus Oesophagealdrüsenzellen von Rana esculenta. Osmiumsäure, Hämatoxylin. Zeiss F, Oc. III.

Fig. 51. Drüsenzellenpaar ans der Saugscheibe von Argulus foliaceus. Die einzelnen Zellen sind in ihren Details nicht gleichzeitig gezeichnet; die untere mit mononucleolärem Kern am 12. October, die obere am 18. October 1881. Die weiteren Veränderungen sind in Figg. 40 und 41 dargestellt. Alle diese vier Zeichnungen stammen von demselben Drüsenzellenpaar. a vermuthlicher Ausführungsgang, $g$ Strahlenfigur.

Fig. 52. Aus dem Oesophagus von Rana esculenta; 3 Wochen Hunger während des Winters. b polynucleolärer Kern aus demselben Präparat.

Fig. 53. Aus dem Oesophagus von Rana eseulenta 71 Stunden nach der Fütterung; Osmiumsäure, Alcohol, Hämatoxyltn. Zeiss CC, Oc. III. n Nebenkern.

Fig. 54. Frisch in Jodserum isolirte Zelle des Pancreas von Salamandra maculosa, 10\% Tage nach der Fütterung. Die Kernsubstanz ist wurstförmig an der Kcrnwand ausgebreitet. Zeiss F, Oc. II.

Fig. 55. Aus dem Pancreas von Salamandra maculosa frisch in Humor aqueus. n Nebenkern.

Fig. 56. Ebendaher; zwei Nebenkerne in der Zelle; links davon ein isolirter Nebenkern.

Fig. 57. Aus dem Pancreas von Salamandra maculata frisch in Jodserum zerzupft. Die farblose Kugel am Kern zeigt sich auch in Zellen, die in Humor aqueus untersucht werden, tritt nicht an allen Zellen auf und bleibt bei Tinktionen farblos.

Fig. 58. Isolationspräparat aus dem Pancreas von Salamandra maculoso nach Behandlung mit Chromsäure, Alcohol und Safranin. n Nebenkern solid in einer Vacuole gelegen. NB. In frischen Isolationspräparaten sieht man viele kleine Kugeln von der Grösse dieses Nebenkernes im Präparat flottiren.

Fig. 59. Ein gleiches Präparat wie das vorige; n Nebenkern aus zwei soliden Körperchen bestehend und in einer Vacuole gelegen.

Fig. 60. Eine isolirte Zelle aus dem Pancreas von Salamandra maculosa mit buchtigem Kern. (Das Thier hatte längere Zeit vorher gehungert.) 
C. Nörner: Beitrag zur Behandlung mikroskopischer Präparate. 351

Fig. 61. Unteres Bruchstück einer pancreatischen Zelle von Salamandra maculosa mit knäuelförmigem breitfadigem Nebenkern. Zeis F, Oc. III. n Nebenkern.

Fig. 62. Eine pancreatische Zelle von Salamandra maculosa mit spiralig gedrehtem Nebenkern $n$.

Fig. 63. Frisch in Jodserum isolirte Formen von Nebenkernen.

Fig. 64-70. Kernformen aus dem Ueberzug der Leber von Salamandra maculosa.

Fig. 64-67. Eingeschnürte und multiple Kerne in den Zellen.

Fig. 68-70. Progressive Verdickung und Ausbildung des Fadenapparates in den Kernen. (NB. $\mathrm{Da}$ es hier nur darauf ankommt, das Vorhandensein und das Fehlen von feinen Netzen in den Kernen zu illustriren, so sind die bekannten, in unserm Object in grosser Zahl nebeneinander vorkommenden, weiteren Stadien der indirecten Kerntheilung nicht abgebildet. Nochmals sei aber darauf hingewiesen, dass der in $0,2 \%$ Chromsäure gehärtete Leberüberzug von Salamandra maculosa vorzugsweise zur Demonstration der verschiedenen Phasen der indirecten Kerntheilung sich eignet.)

\section{Beitrag zur Behandlung mikroskopischer Präparate.}

Von

\section{Dr. C. Nörner.}

(Wiener K. K. Thierarznei-Instilut.)

Die Haupteinbettungsflüssigkeiten für mikroskopische Präparate, die gegenwärtig in der mikroskopischen Technik Verwendung finden, sind: der Canadabalsam, der Damarlack und das Glycerin. Der Canadabalsam leidet an dem Febler, dass er meistens zu gelb ist, mit Ausnahme des englischen, welcher wohl der beste, aber in Deutschland schwer zu bekommen ist. Der Damarlack, welcher frïher mehrfach verwendet wurde, von dem Canadabalsam aber 